\title{
Mating-Induced Expression of c-fos in the Male Syrian Hamster Brain: Role of Experience, Pheromones, and Ejaculations
}

\author{
Sara Kollack-Walker, Sarah W. Newman \\ Department of Anatomy and Cell Biology, 5710 Medical Science II Building, \\ University of Michigan, Ann Arbor, Michigan 48109-0616
}

Received 2 August 1996, accepted 20 December 1996

\begin{abstract}
This study was designed to investigate the effects of pheromonal cues and specific behaviors within the male copulatory sequence on c-fos expression in the medial nucleus of the amygdala (Me), the bed nucleus of the stria terminalis (BNST), and the medial preoptic area (MPOA) of the Syrian hamster brain. Sexually experienced male hamsters were placed into clean testing arenas and were either: 1) left alone as handled controls; 2) exposed to female hamster vaginal secretion (FHVS) on cotton swabs; or mated to various end points of copulation with a sexually receptive female: 3 ) five intromissions, 4 ) one ejaculation, 5) five ejaculations, or 6) long intromissions. A seventh group of sexually naive control males 7) was left alone in the arena. The brains of these males were compared to those of the sexually experienced controls to determine whether exposure to cues associated with prior sexual experience could alter cfos expression. In males exposed only to FHVS, Fos immunoreactivity (Fos-ir) increased within the posterodorsal Me, the anterodorsal part of the posterome-
\end{abstract}

dial BNST, and the magnocellular medial preoptic nucleus (MPNmag). Following one ejaculation, Fos-ir increased within the caudal posterodorsal $\mathrm{Me}$, the dorsolateral MPOA, and the paraventricular nucleus of the hypothalamus. After multiple ejaculations, additional labeling was observed within the posteroventral part of the posteromedial BNST, the medial preoptic nucleus (MPN), the central tegmental field, and in cell clusters of the caudal posterodorsal Me and rostral posteromedial BNST. Fos-ir also increased within the posterodorsal Me, MPN, and MPNmag in sexually experienced control males exposed to the empty test chamber compared to sexually naive males exposed to an identical chamber. These results demonstrate that the mating-induced pattern of neuronal activation in sexually experienced males is dependent upon multiple factors, including prior sexual experience in the testing environment, investigation of FHVS, and the number of ejaculations achieved. (ㄷ) 1997 John Wiley \& Sons, Inc. J Neurobiol 32: 481-501, 1997 Keywords: copulation; chemoinvestigation; experience; c-fos; limbic

\section{INTRODUCTION}

Mating behavior induces c-fos expression within the male Syrian hamster brain (Kollack and Newman, 1992; Fernandez-Fewell and Meredith, 1994; Kollack-Walker and Newman, 1995 ). In particular,

\footnotetext{
Correspondence to: S. W. Newman

Contract grant sponsor: NIH-NICHD Center for the Study of Reproduction at University of Michigan; contract grant numbers: NS20629, P30-HD-18258, DA07268-04

(C) 1997 John Wiley \& Sons, Inc. CCC 0022-3034/97/050481-21
}

selective and significant increases in the number of Fos-immunoreactive (Fos-ir) neurons are observed within subnuclei of the medial amygdala, the bed nucleus of the stria terminalis (BNST), and the medial preoptic area (MPOA), three brain areas where lesions eliminate or alter male hamster sexual behavior (Lehman et al., 1980, 1983; Lehman and Winans, 1982; Powers et al., 1987). The expression of the c-fos proto-oncogene has been correlated with neuronal activation (Hunt et al., 1987; Morgan et al., 1987; Sagar et al., 1988; Dragunow and Faull, 1989 ); thus, the selectively increased production of 
Fos protein within these groups of neurons following mating is interpreted to result from neuronal activation associated with this behavior.

The pattern of mating-induced c-fos expression reflects, in part, the processing of female pheromones. Female hamster vaginal secretion (FHVS) contains pheromones that stimulate sexual attraction and arousal (Murphy, 1973; Johnston, 1974, 1975; Darby et al., 1975), and exposure of male hamsters to this secretion results in increased Fos production in neurons within the medial nucleus of the amygdala, BNST, and MPOA (Fiber et al., 1993; Fernandez-Fewell \& Meredith, 1994). However, the neuroanatomical pattern of activation produced by FHVS exposure represents only a portion of the activation observed within these three areas following mating (Kollack and Newman, 1992; Fernandez-Fewell and Meredith, 1994; Kollack-Walker and Newman, 1995) suggesting that mounts, intromissions, or ejaculations are also involved in the development of the postmating pattern.

In other species sensory information associated with intromissions (Baum and Everitt, 1992) or ejaculations (Coolen, 1995; Heeb and Yahr, 1996) has been implicated in the production of Fos protein observed following mating. However, the relationship between the anatomical distribution of neuronal activation and the occurrence of specific components of copulation, such as intromissions or ejaculations, has not been delineated in the hamster.

In addition, experiential factors may influence cfos expression. The effects of conditioned stimuli on c-fos induction have been observed following foot-shock conditioning (Pezzone et al., 1992a, 1992b), taste aversion learning (Swank and Bernstein, 1994), and environmental conditioning (Brown et al., 1992). Although sexual arousal (Zamble et al., 1985, 1986; De Jonge et al., 1992) and androgen surges (Kamel and Frankel, 1978; Graham and Desjardins, 1980) do occur in response to conditioned stimuli, the neuroanatomical pattern of Fos immunoreactivity associated with such responses has not been studied.

In the study reported here, the effect of mating to different behavioral end points on Fos immunostaining in the medial amygdala, BNST, MPOA, hypothalamus, and central tegmental field was determined in the male Syrian hamster brain. On the day of the experiment six groups of sexually experienced adult male hamsters were placed into clean, empty testing arenas, where they were left alone as handled controls, were exposed to FHVS on cotton swabs, or were mated to one of several end points of copulation with a sexually receptive female: five intromissions, one ejaculation, five ejaculations, or long intromissions. Unlike the short intromissions preceding each ejaculation, in which the male mounts the female, intromits, and quickly dismounts, long intromissions are associated with the onset of sexual satiety (Bunnell et al., 1977). During this behavior, the male mounts the female and intromits, but instead of dismounting quickly, he maintains vaginal penetration and shows a repetitive thrusting pattern (Bunnell et al., 1977). A seventh group of sexually naive males was included to determine if prior sexual experience in the testing arena had an affect on c-fos expression in response to being placed in the arena.

\section{MATERIALS AND METHODS}

\section{Behavioral Testing}

Adult male Syrian hamsters (Mesocricetus auratus; Charles River, Kingston, NY) were group-housed under a long photoperiod (14:10 h light/dark cycle) with food and water ad libitum. A total of 43 male hamsters were used in this study. Thirty-six of these animals were given sexual experience by allowing each male to mate with a sexually receptive female on three separate occasions. Sexual receptivity was induced in adult ovariectomized female hamsters by subcutaneous injections of $10 \mu \mathrm{g}$ estradiol benzoate 48 and $24 \mathrm{~h}$ prior to, and $250 \mu \mathrm{g}$ progesterone $4-6 \mathrm{~h}$ before testing. During the first $6 \mathrm{~h}$ of the dark cycle (between 13:00 and 19:00 h), each male was placed in a testing arena on a dimly lit table or floor and allowed to interact with a receptive female until the male achieved an ejaculation or $15 \mathrm{~min}$ had elapsed. The testing arena was a transparent plastic cage containing bedding, and the testing arena was used for several sexual experience tests. The female was placed into the testing arena immediately after placement of the male. Each male used in this study achieved an ejaculation on at least two of the three pretests. Following the pretests, these males were randomly assigned to one of six different groups of six males each: 1) handled controls (HC-EXP), 2) FHVS, 3) mated to five intromissions (5-INTRO), 4) mated to one ejaculation (1-EJAC), 5) mated to five ejaculations (5-EJAC), and 6) mated to long intromissions (long-INTRO). As described above, long intromissions, consisting of repetitive thrusting upon vaginal penetration but without ejaculation, occur at the culmination of a mating sequence following multiple ejaculations. The number of ejaculations achieved by the males in the longINTRO group in this study varied from six to 10 .

The remaining seven males were used to determine if prior sexual experience in the testing arena influenced cfos expression. Each male in this group, the HC-NAIVE group, was placed into a clean (odor-free) testing arena with bedding and left alone for $10 \mathrm{~min}$ on each of the three separate pretest occasions, while the other males were acquiring sexual competence. This handling was 
Table 1 Summary of Copulatory Behavior Shown by Males in This Study

\begin{tabular}{lccrr}
\hline & & \multicolumn{3}{c}{ Copulatory Behavior } \\
\cline { 2 - 5 } \multicolumn{1}{c}{ Groups } & $\begin{array}{c}\text { Mounts } \\
\text { Intromissions }\end{array}$ & Intromissions & Ejaculations & Time (s) \\
\hline 5-INTRO & $2.5 \pm 0.5$ (range: $2-3)$ & 5 & 0 & $149 \pm 27$ \\
1-EJAC & $4.0 \pm 1.2$ (range: $2-6)$ & $12 \pm 3.0$ (range: $4-21)$ & 1 & $239 \pm 46$ \\
5-EJAC & $13.0 \pm 4.5$ (range: $7-26)$ & $25 \pm 5.3$ (range: $9-46)$ & 5 & $678 \pm 80$ \\
Long-INTRO & $8.0 \pm 1.8$ (range: $3-13)$ & $26 \pm 4.1$ (range: $14-43)$ & $8 \pm 1.0$ (range: $6-10)$ & $851 \pm 53$ \\
\hline
\end{tabular}

${ }^{1}$ Number of males displaying mounts without intromission: 5-INTRO $(n=2), 1$-EJAC $(n=3), 5$-EJAC $(n=4)$, and Long-INTRO $(n=5)$.

designed to ensure that the only difference between the HC-EXP and HC-NAIVE groups on the day of the experiment (described below) was whether a given male had mated with a receptive female during the pretests.

On the day of the experiment, behavioral tests were conducted within the first $6 \mathrm{~h}$ of the dark cycle. During testing, each male was taken from his home cage and placed into a clean (odor-free) testing arena located on a dimly lit table. The male then was either left alone for 10 min (HC-NAIVE and HC-EXP), given two cotton swabs containing FHVS in succession over a 10 -min period (FHVS), or given access to a sexually receptive female and allowed to mate with her to 5-INTRO, 1EJAC, 5-EJAC, or to long-INTRO. The female was placed into the testing arena immediately after placement of the male. Although the duration of interaction with the female varied in these latter groups (Table 1), previous work in our laboratory has determined that it is the type of behavior shown and not the length of time spent in the presence of a receptive female that is critical for the production of Fos protein following mating (unpublished observations). Following testing, each male was left alone in the testing arena with food and water.

\section{Tissue Processing}

One hour after the onset of behavioral testing, each male was deeply anesthetized with sodium pentobarbital (12 $\mathrm{mg} / 100 \mathrm{~g}$ body wt) and perfused through the aorta with $50 \mathrm{~mL}$ phosphate-buffered saline $(0.1 \mathrm{M}$ sodium phosphate buffer, $0.9 \%$ sodium chloride, and $0.1 \%$ sodium nitrate as a vasodilator) at room temperature, followed by $200 \mathrm{~mL} 4 \%$ paraformaldehyde in $0.1 \mathrm{M}$ sodium phosphate buffer at $4^{\circ} \mathrm{C}$. The brain was removed from the skull and cut into $40-\mu \mathrm{m}$ coronal sections, and a series of sections spaced approximately $80 \mu \mathrm{m}$ apart was processed for Fos immunocytochemistry as previously described (Kollack and Newman, 1992). Visualization of the Fos antigen was accomplished using the avidin-biotin-peroxidase complex technique (Hsu et al., 1980) with nickel chloride-enhanced diaminobenzidine tetrahydrochloride as the chromagen (Hsu and Soban, 1982).

The primary antiserum was a polyclonal rabbit anti- c-fos antiserum (Biotechnology, Inc., Santa Cruz, CA, Cat. No. sc-52, Lot No. G152; final dilution 1:1000) directed against amino acid residues 3-16 of the human Fos protein (van Straaten et al., 1983). To test for nonspecific staining that may be associated with the Santa Cruz c- fos antiserum or the secondary antiserum [biotinylated donkey anti-rabbit immunoglobulin $\mathrm{G}$ ( $\mathrm{IgG})$, Jackson ImmunoResearch Laboratories, Inc., West Grove, PA] in hamster brain, sections from a 5-INTRO male were processed as previously described following: 1 ) preincubation of the primary antiserum with Fos peptide (Santa Cruz, Cat No. sc-52P) for $2 \mathrm{~h}$ at room temperature, or 2) omission of the primary antiserum. An additional series of sections from the same 5-INTRO male was processed using the standard immunocytochemical protocol for comparison to the preabsorption and secondary-only controls. The concentration of the Fos peptide used in the preabsorption step was 10 times higher than the concentration of the Fos antiserum.

To determine if the Santa Cruz c- fos antiserum detects similar levels of basal Fos expression as the Oncogene Sciences Ab-2 antiserum reported previously (Kollack and Newman, 1992; Kollack-Walker and Newman, 1995) one series of sections from an HC-NAIVE male was processed using the Santa Cruz antiserum and an adjacent series using the Oncogene Sciences antiserum.

\section{Data Analysis}

The magnitude of neuronal activation was determined by counting Fos-ir cells within specific subnuclei at a magnification of $\times 200$ with the aid of an optical reticle. All slides were coded and cell counts were performed with the observer blind to the experimental group of the animal. The selection of sections to be used for cell counts was based on specific neuroanatomical landmarks and optimal Fos immunostaining. For all but one of the brain areas studied, two cell counts were taken for each subnucleus - one from each hemisphere of the brain - and averaged to provide 1 data point/subnucleus per brain. These data points were then averaged within behavioral groups to determine the mean number of Fos-ir neurons per subnucleus per group. The single exception to this 

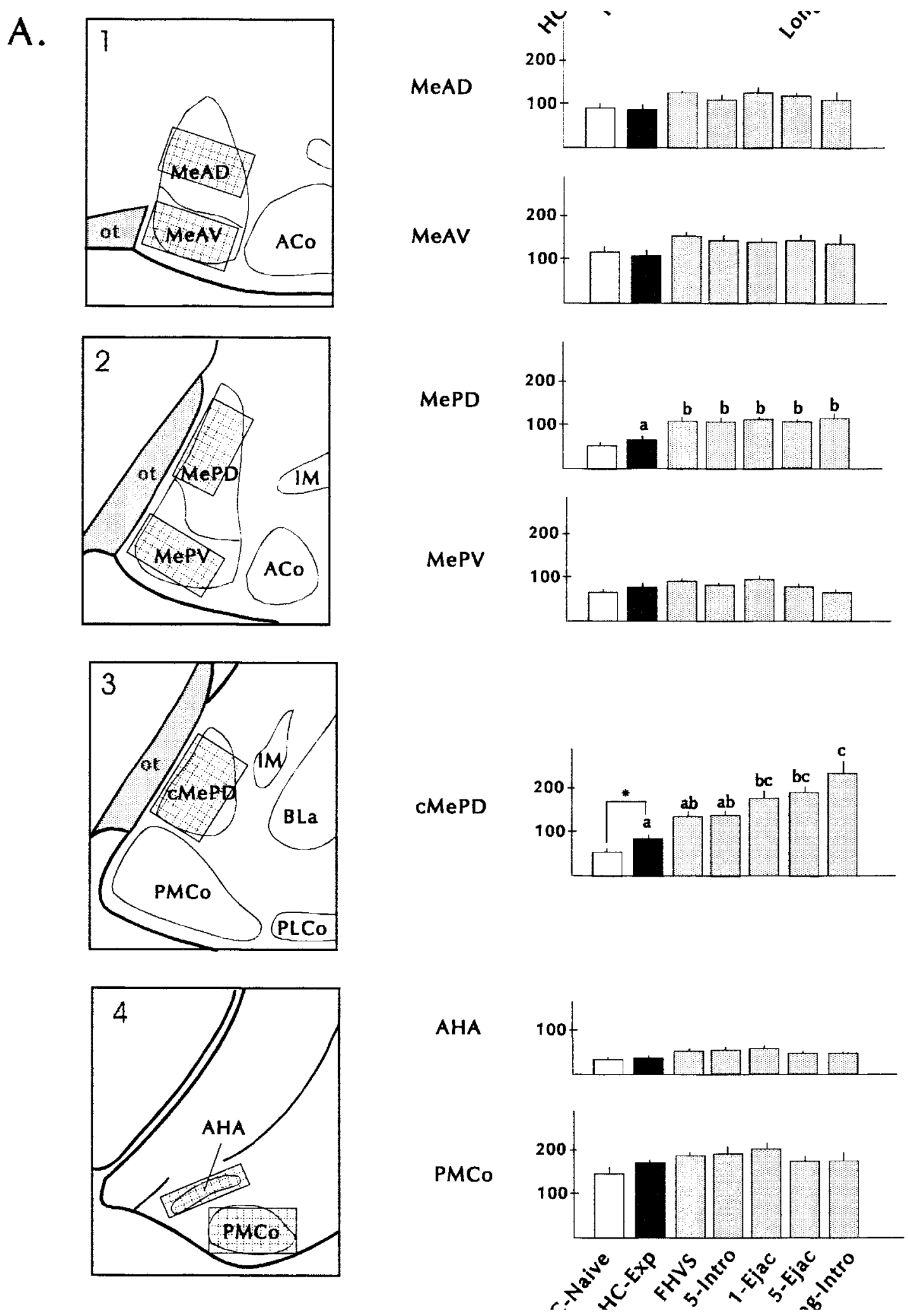

Figure 1 The location and area of Fos-ir cell counts within the medial amygdala (A1-4), BNST (B1-4), MPOA (C1-3), and hypothalamus and midbrain CTF (D1-4), and the resultant mean number of Fos-ir neurons (S.E.M.) per nucleus or subnucleus for HC-NAIVE, HC-EXP, FHVS, 5-INTRO, 1-EJAC, and Long-INTRO groups. A significant increase in the 
B.
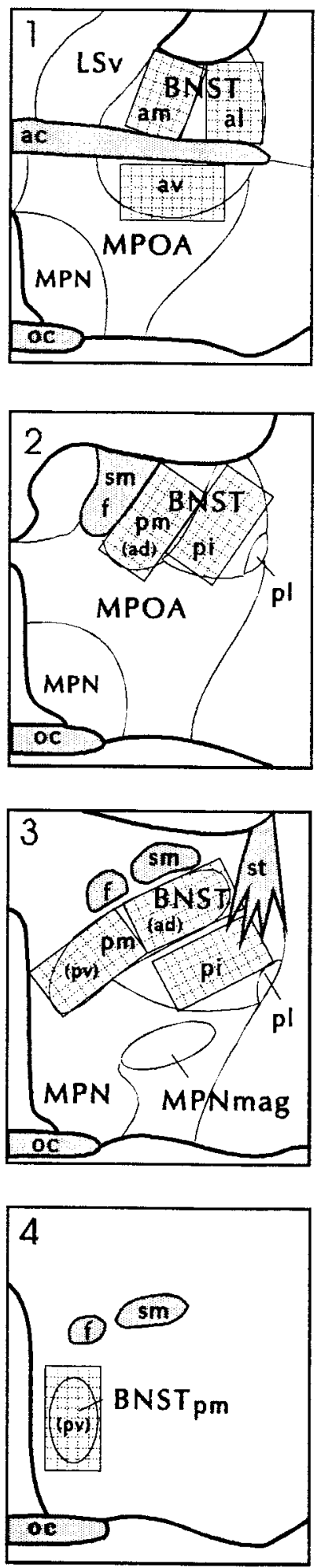

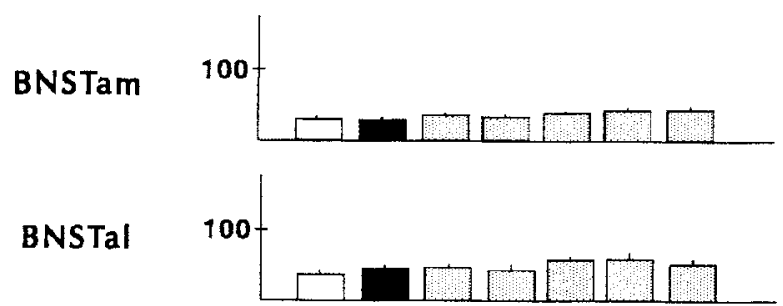

BNSTav

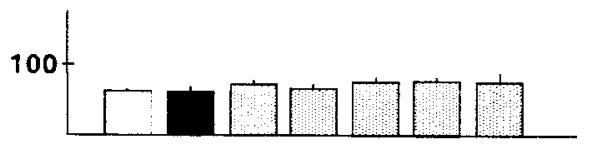

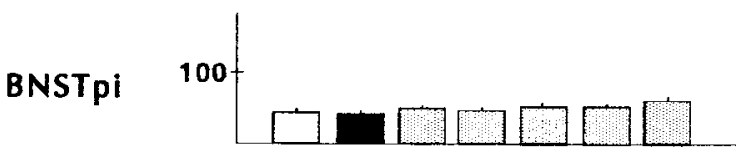

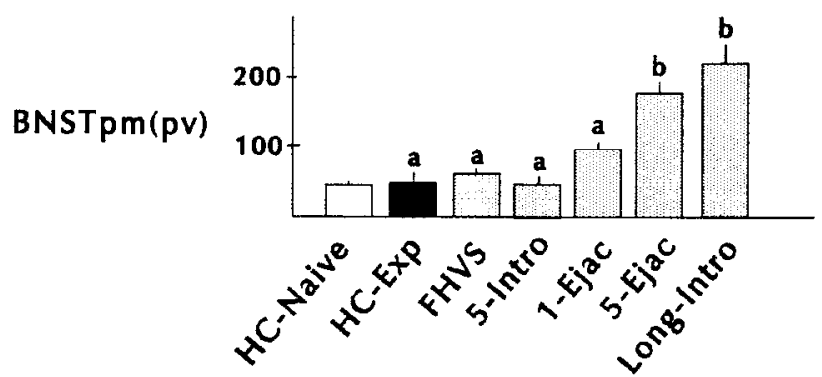

number of Fos-ir neurons in HC-EXP group above the HC-NAIVE group is indicated with an asterisk $(p<0.05)$. Brain regions in which a significant difference was detected among the HC-EXP, FHVS, 5-INTRO, 1-EJAC, 5-EJAC, and Long-INTRO groups are identified by lowercase letters above each bar $(p<0.05)$. The statistical relationship between the groups is indicated by lowercase letters; groups that share a common letter do not differ. 

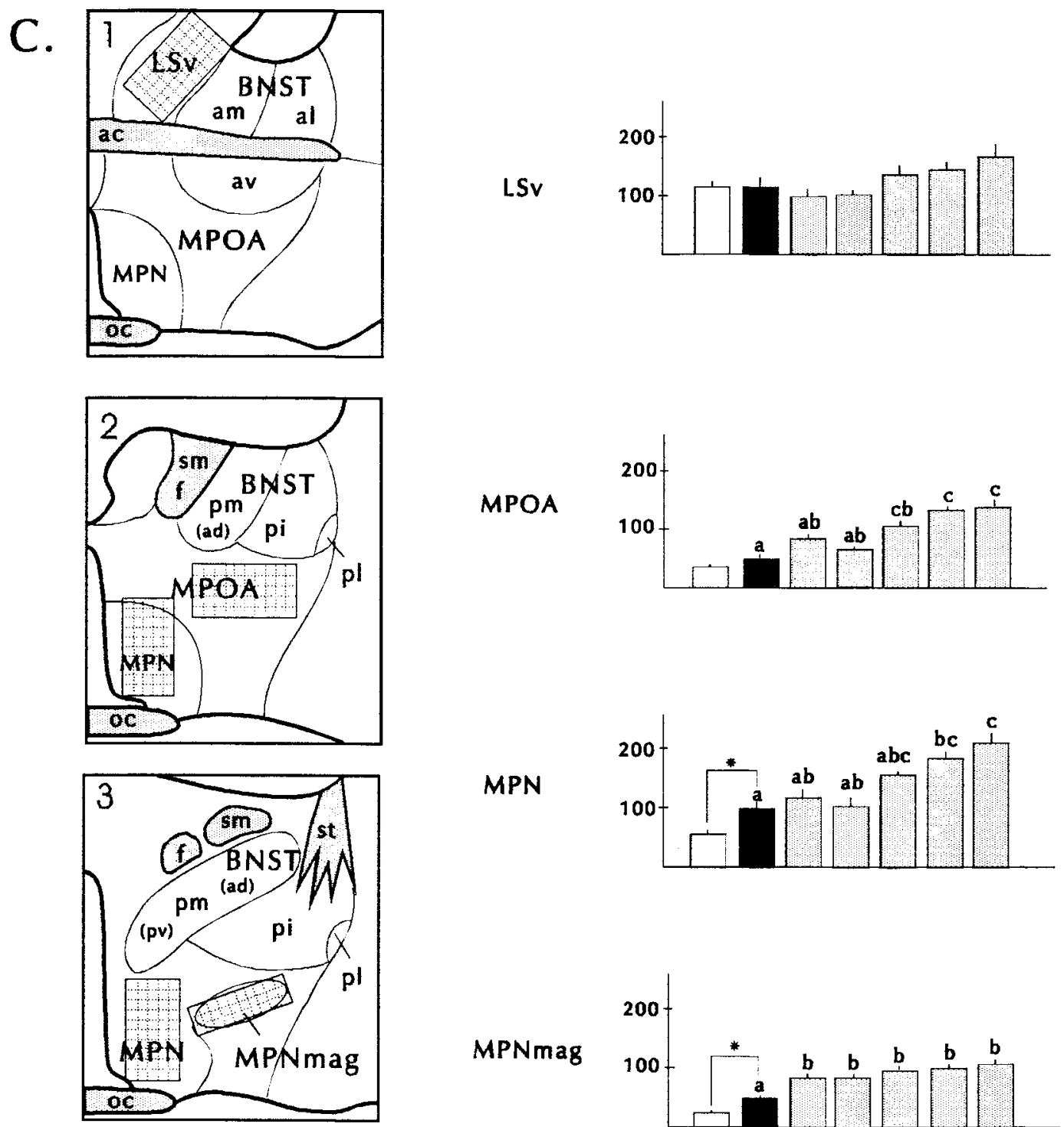

MPN
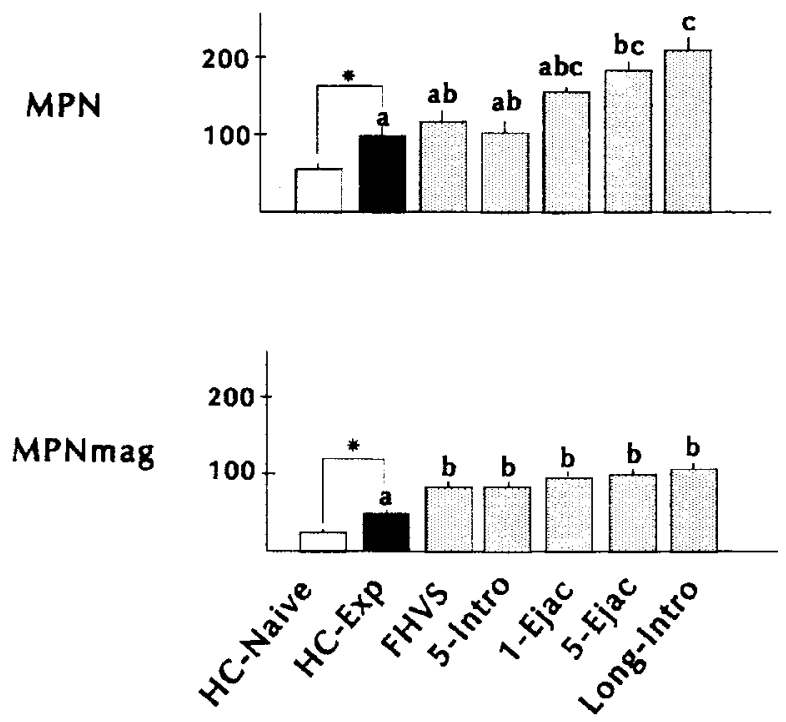

Figure 1 (Continued)

design was the analysis of the medial preoptic nucleus (MPN), in which three cell counts were taken on both sides of the brain and the resulting six values were averaged to provide 1 data point/MPN per brain. Multiple cell counts within this subnucleus were necessary because the size of this nuclear area and slight variations in the angle of sectioning made it impossible to use surrounding landmarks to consistently select precisely the same level at which to take only one sample of Fos immunostaining. The size of the area sampled for each nucleus varied and was determined by the size and shape of the nucleus: ventral premammillary nucleus (PMV) $\left(0.105 \mathrm{~mm}^{2}\right)$, amygdalohippocampal area (AHA) and magnocellular MPN (MPNmag) $\left(0.126 \mathrm{~mm}^{2}\right)$, bed nucleus of stria terminalis, anteromedial (BNSTam) and BNST, anterolateral (BNSTal) $\left(0.168 \mathrm{~mm}^{2}\right)$, paraventricular nucleus of the hypothalamus $(\mathrm{PVN})\left(0.231 \mathrm{~mm}^{2}\right)$, caudal extension of the posterodorsal medial nucleus of the amygdalal (cMePD) $\left(0.294 \mathrm{~mm}^{2}\right)$, and all other subnuclei $(0.210$ $\mathrm{mm}^{2}$ ). The location and size of each area analyzed are illustrated in Figure 1, where the size and shape of the reticle configuration used for counting is superimposed over a drawing of each nucleus.

A one-factor analysis of variance was used to com- 

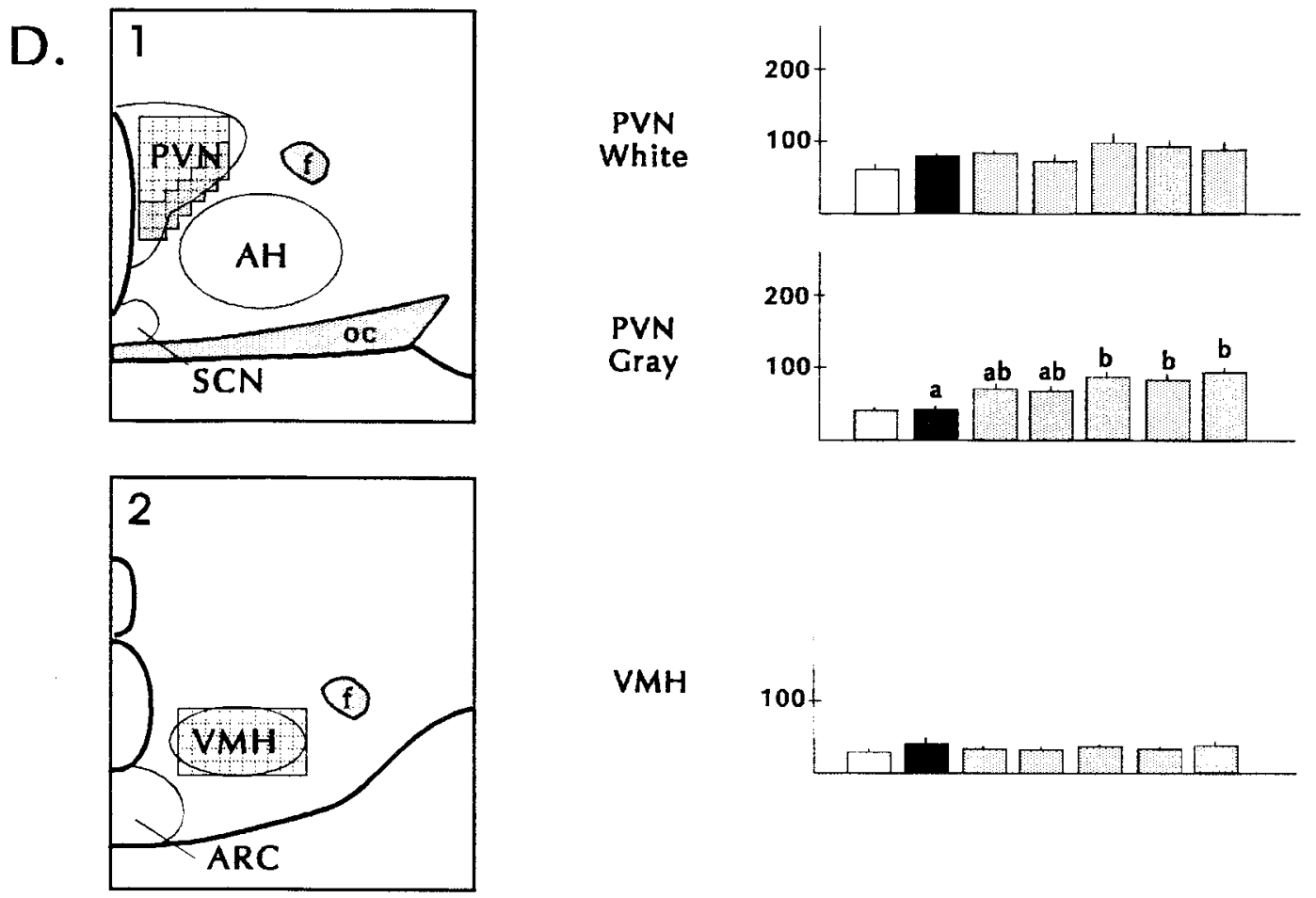

VMH
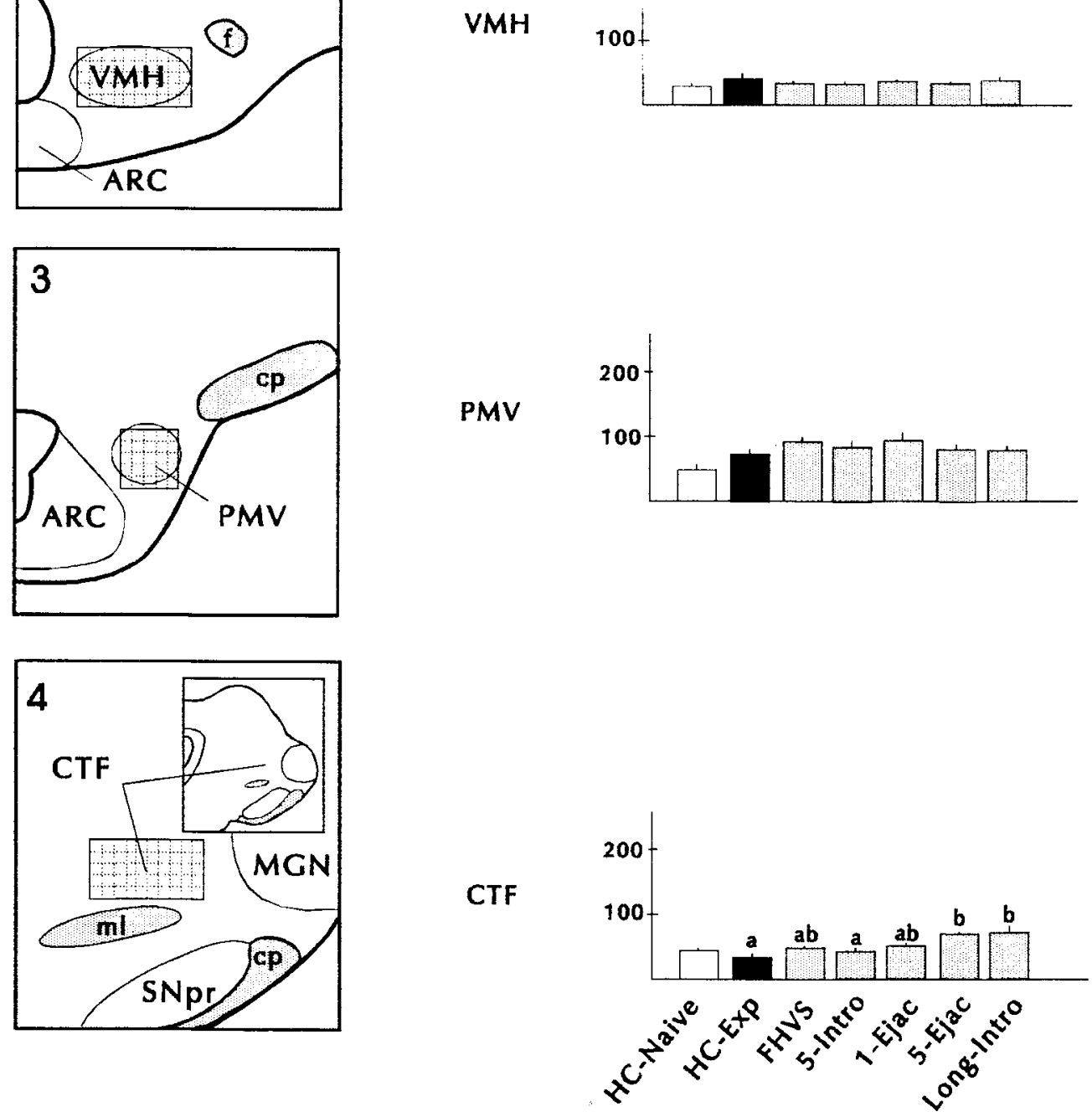

Figure 1 (Continued)

pare the mean number of Fos-ir neurons per brain area among HC-EXP, FHVS, 5-INTRO, 1-EJAC, 5-EJAC, and Long-INTRO groups. Post hoc comparisons were based on the Tukey test using a 0.05 level of confi- dence. To determine if prior sexual experience in the testing arena induced c-fos expression within any of these brain regions on the day of testing, Students $t$ tests (two-tailed, $p<0.05$ ) were used to compare the 
mean number of Fos-ir neurons between HC-NAIVE and HC-EXP groups.

\section{RESULTS}

\section{Behavioral Observations}

A summary of the copulatory behavior shown by males who interacted with sexually receptive females is provided in Table 1 . The most variable aspect of the males' behavior was mounts without intromissions; only a subset of the males in each group showed this response. The remainder of the males intromitted on the first and all subsequent mounts. As expected, males who mated to multiple ejaculations took longer to do so and required greater numbers of total intromissions than males who mated to only one ejaculation. Although males in the Long-INTRO group achieved on average eight ejaculations compared to the five ejaculations achieved by males in the 5-EJAC group, there was no difference in the total number of intromissions achieved by males in these two groups.

\section{Immunocytochemical Controls}

Preincubation of the primary antiserum with an excess of the Fos peptide or omission of the primary antiserum eliminated all Fos immunostaining. No differences were observed in the pattern or overall level of c-fos expression in two adjacent series of sections immunostained with the Santa Cruz and Oncogene Sciences c-fos antisera.

\section{Fos Expression}

Overview. Figure 1 summarizes the effect of sexual experience, female vaginal odors, and mating to different end points of copulation on the magnitude of c-fos expression within nuclei and subnuclei of the medial amygdala [Fig. 1(A)], BNST [Fig. 1(B)], MPOA and septum [Fig. 1(C)], and hypothalamus and midbrain CTF [Fig. 1(D)]. The results demonstrated a significant increase in the number of Fosir neurons within specific subdivisions of the medial nucleus of the amygdala (Me) and MPOA in sexually experienced control males compared to the naive controls, when males in both groups were placed in an empty testing arena on the day of the experiment. In addition, in sexually experienced males, Fos immunostaining increased within discrete subdivisions of the Me, posteromedial BNST, and MPOA following exposure to FHVS or mating to one or more ejaculations. Mating to ejaculation also produced activation within the paraventricular nucleus of the hypothalamus and within the midbrain CTF. However, mating to only five intromissions did not alter the number of Fos-positive cells in any of the brain areas studied.

Medial Amygdala. A significant increase in the number of Fos-ir neurons was observed in MePD in males exposed to FHVS compared to HC-EXP males. Although in the caudal extension of this brain area (cMePD) the absolute number of Fos-ir neurons did not differ between FHVS and HC-EXP males, a qualitative difference was observed. Fospositive neurons were seen adjacent to the optic tract in this region in the FHVS males but not in HC-EXP controls (data not shown).

In $\mathrm{cMePD}$, the data revealed two additional characteristics of mating-related neuronal activation. First, the total number of Fos-ir neurons was significantly elevated in the HC-EXP group compared to the HC-NAIVE group even though neither group of males had been paired with a female on the day of the experiment [Fig. 2(A,B)]. Second, although no difference was detected in the mean number of Fos-ir neurons in cMePD among the 1-EJAC, 5EJAC, and Long-INTRO groups, only males in the 5-EJAC and Long-INTRO groups showed a selective pattern of Fos immunostaining within dorsal and lateral cell clusters in cMePD (Fig. 3 ). In addition, the appearance of these clusters seemed to be influenced by the number of ejaculations achieved, as only $67 \%$ (four of six) of the males achieving five ejaculations had these cell clusters, while they were present in $100 \%$ ( $\operatorname{six}$ of six) of the males achieving long intromissions.

Bed Nucleus of the Stria Terminalis. Significant increases in the number of Fos-ir neurons above the HC-EXP group were observed throughout the posteromedial subdivision of the BNST (BNSTpm), with the specific pattern of activation dependent upon the endpoint of mating [Figs. 1(B) and 4]. The posteromedial BNST has been subdivided into anterodorsal [BNSTpm(ad)] and posteroventral [BNSTpm(pv)] levels based on the pattern of neuronal activation following sexual or agonistic stimuli (Kollack-Walker and Newman, 1995). In the present study, Fos expression increased within BNSTpm(ad) of males exposed to FHVS, with further increases seen in males who copulated to multiple ejaculations. BNSTpm(ad) corresponds to the caudal posterior medial BNST (mBNSTpc) described by Fernandez-Fewell and Meredith (1994). In BNSTpm(pv), Fos production 

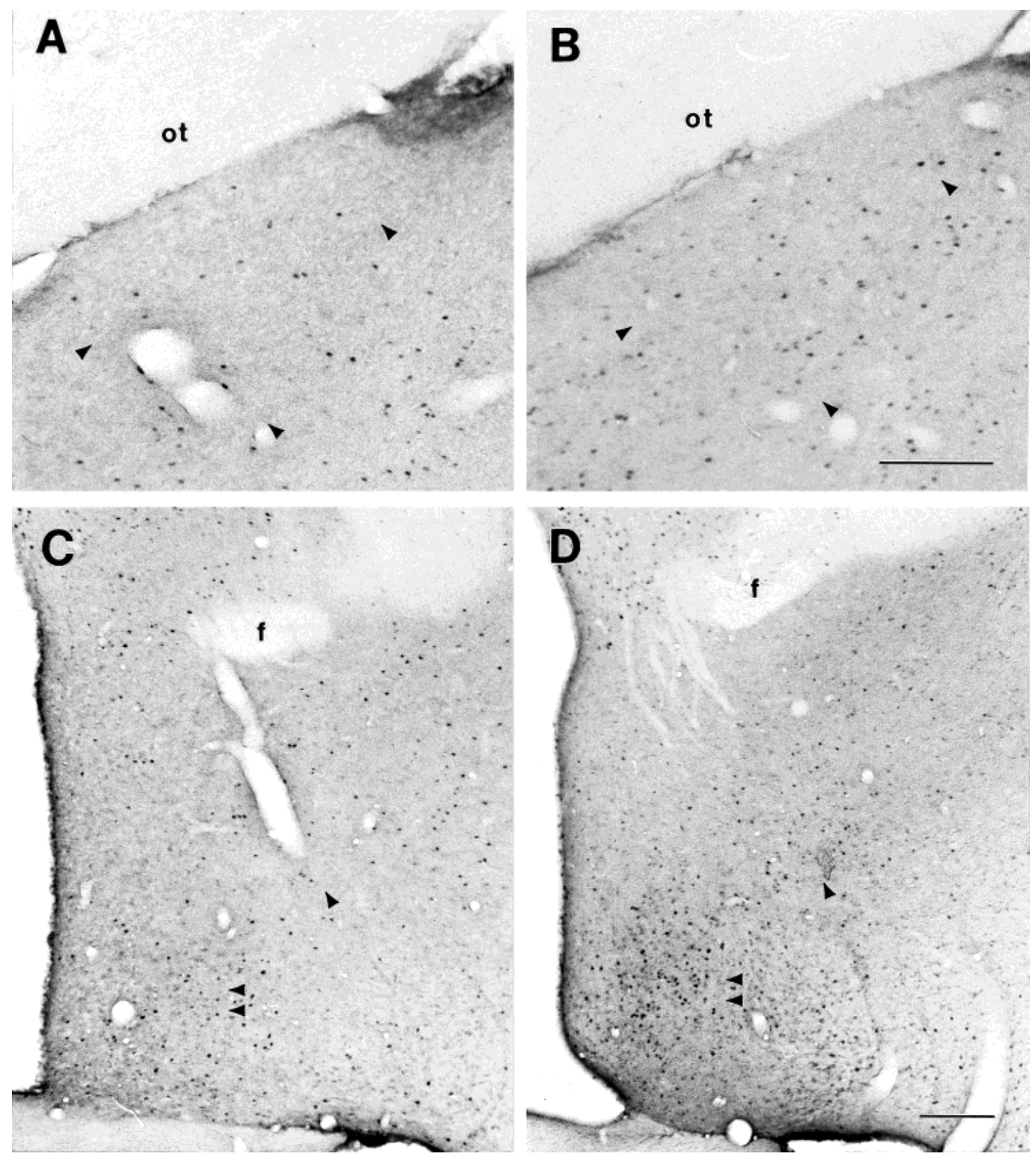

Figure 2 Photomicrographs of Fos-immunostaining in $\operatorname{HC}-N A I V E(A, C)$ and $\operatorname{HC}-E X P(B, D)$ males in cMePD [(A,B); see Fig. 1 (A3) for orientation to the photomicrographs] and MPOA [(C,D); see Figs. 1(B3) and 4(A) for orientation to the photomicrographs]. Note the increase in the number of Fos-ir neurons in the experienced males in cMePD, MPN, and MPNmag. In $(A, B)$, the single arrowheads indicate the corners of the triangular-shaped cMePD. In (C,D) the single arrowheads identifies the lateral border of MPNmag, and the double arrowheads identify the lateral border of MPN. Scale bar $=200 \mu \mathrm{m}$.

increased only in males who mated to five or more ejaculations.

Copulation-specific cell clusters were also observed within the posteromedial BNST, at a level rostral to BNSTpm(ad). These clusters, originally described by Fernandez-Fewell and Meredith (1994) as lying within the rostral posterior medial BNST (mBNSTpr), were detected only 


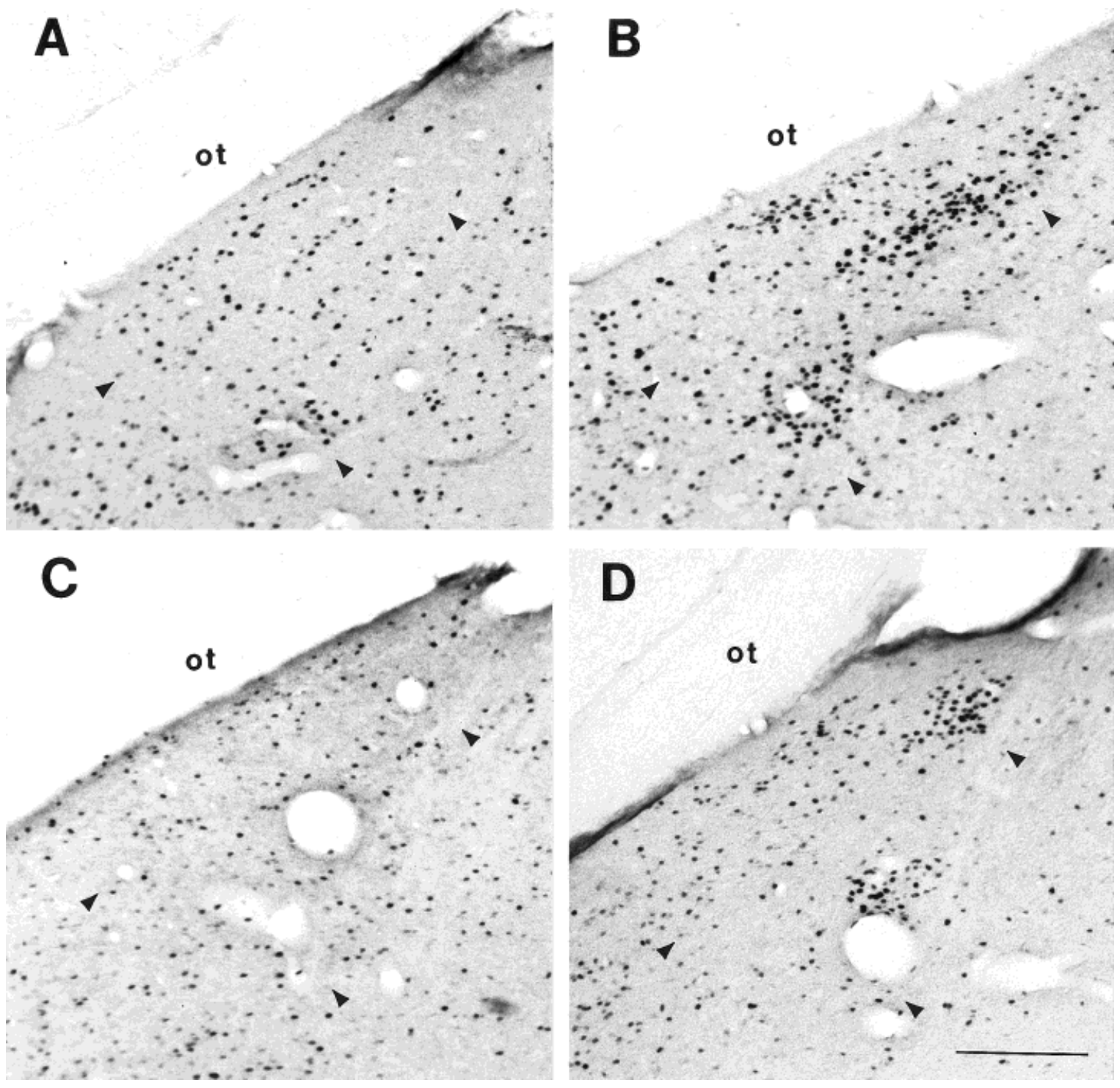

Figure 3 Photomicrographs of Fos-immunostaining in 1-EJAC (A,C) and Long-INTRO (B,D) males in cMePD [see Fig. 1(A3) for orientation to the photomicrographs]. Note the Fos-immunostaining in dorsal and lateral cell clusters in Long-INTRO males (B,D); these photomicrographs are taken from four separate animals (A-D). The arrowheads indicate the corners of the triangular-shaped cMePD. Scale bar $=200 \mu \mathrm{m}$.

in males that mated to five or more ejaculations; Fos immunolabeling was not quantified within this region.

Medial Preoptic Area. The number of Fos-positive neurons increased significantly in MPN and MPNmag in HC-EXP males compared to HC-NAIVE males [Figs. 1(C) and 2(C,D)]. In addition, Fos immunostaining was increased further in MPNmag after exposure to female pheromones; comparison of Figures 2(D) and 4(B) illustrates this difference. The number of Fos-ir neurons also increased in
MPN after mating to five or more ejaculations, although the cumulative effect, analyzed at three rostrocaudal levels of this nucleus, is not clearly seen in comparing the photomicrographs in Figures 2(D) and 4(D). Significant increases in the number of Fos-ir neurons above the HC-EXP group were also observed within the dorsolateral MPOA following one ejaculation.

Hypothalamus. Among the hypothalamic nuclei studied in these brains, only the paraventricular nucleus of the hypothalamus showed significant eleva- 

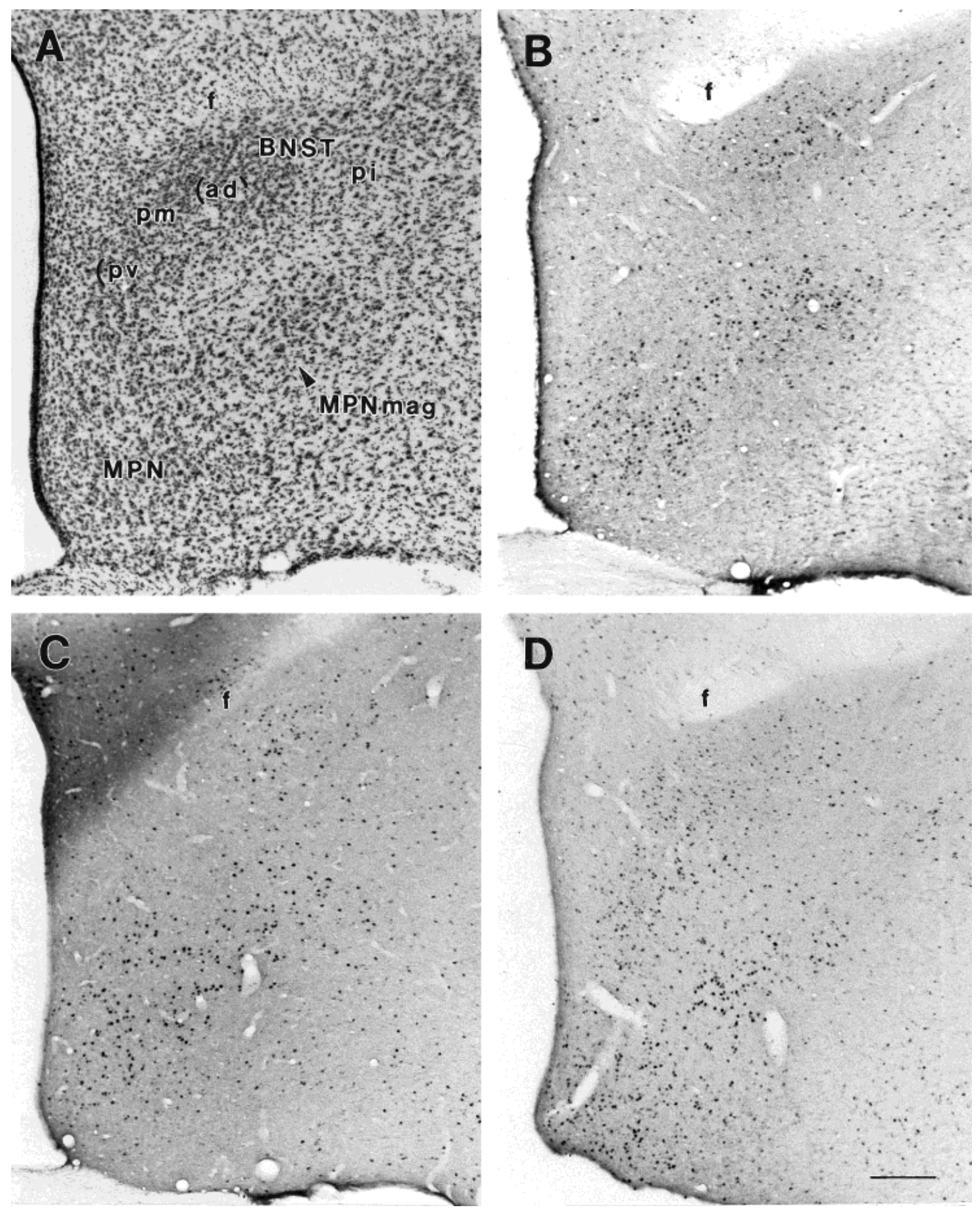

Figure 4 Photomicrographs of a Cresyl violet-stained section, labeled for orientation (A) and Fos-immunostaining (B-D) within specific subdivisions of the BNST and MPOA in FHVS (B), 1-EJAC (C), and Long-INTRO (D) males. For additional orientation to the photomicrographs, see Figure 1(B3). Note Fos-immunoreactive neurons within the BNSTpm $(\mathrm{ad})$ in all males (B-D); an increase in the number of Fos-immunoreactive neurons within BNSTpm(pv) is observed only in Long-INTRO males (D). Scale bar $=200 \mu \mathrm{m}$. 

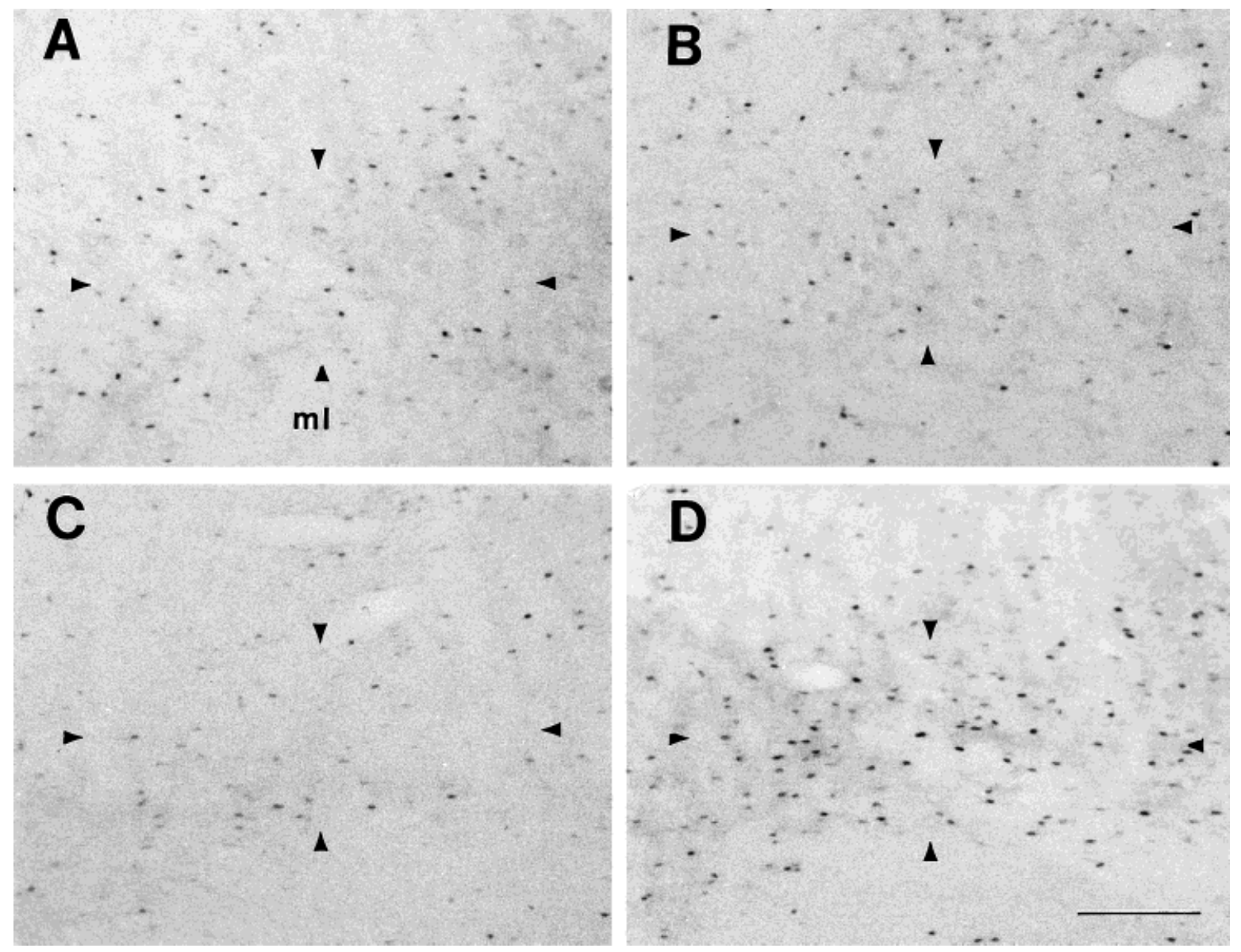

Figure 5 Photomicrographs of Fos-immunostaining within the CTF in HC-EXP (A), FHVS (B), 1-EJAC (C), and Long-INTRO (D) males. At this level within the midbrain, note the significant increase in the number of Fos-immunoreactive neurons immediately dorsal to the medial lemniscus ( "ml"' in A) in Long INTRO males (D). The arrowheads define the borders of the CTF. Scale bar $=100 \mu \mathrm{m}$.

tions in c-fos expression above HC-EXP and this increase occurred following one ejaculation. Further, this effect was localized to the ventrolateral part of the PVN illustrated in gray in Figure 1(D). No effect of sexual experience was observed in any of these hypothalamic nuclei or the midbrain at the levels indicated.

Midbrain. Significant increases in the number of Fos-ir neurons above the HC-EXP group were observed within the central tegmental field following five or more ejaculations (Fig. 5).

Septum. No significant differences above HC-EXP were detected for any of the experimental groups in the ventrolateral septum.

\section{DISCUSSION}

\section{Overview}

The purpose of this study was to identify the specific components of sex behavior that stimulate Fos ex- pression in the brains of male hamsters following mating. The results suggest that select populations of neurons in the amygdala and medial preoptic area can be activated by environmental stimuli previously associated with the experience of mating in a particular setting. Further, the findings presented here support and clarify data from earlier reports of the distinction between areas activated during chemoinvestigatory and those activated by copulatory processes in the male hamster brain.

It is important to acknowledge at the outset that using c- fos expression as a marker of neuronal activation may not identify all neurons that are active during male sex behavior. It is possible that some neurons may not produce Fos protein (possibly using other immediate early gene products), while others with Fos protein may not be visualized and counted. Thus, the important results of this study constitute positive identification of areas that are activated in association with specific behavioral states. 


\section{BNST \& MPOA}
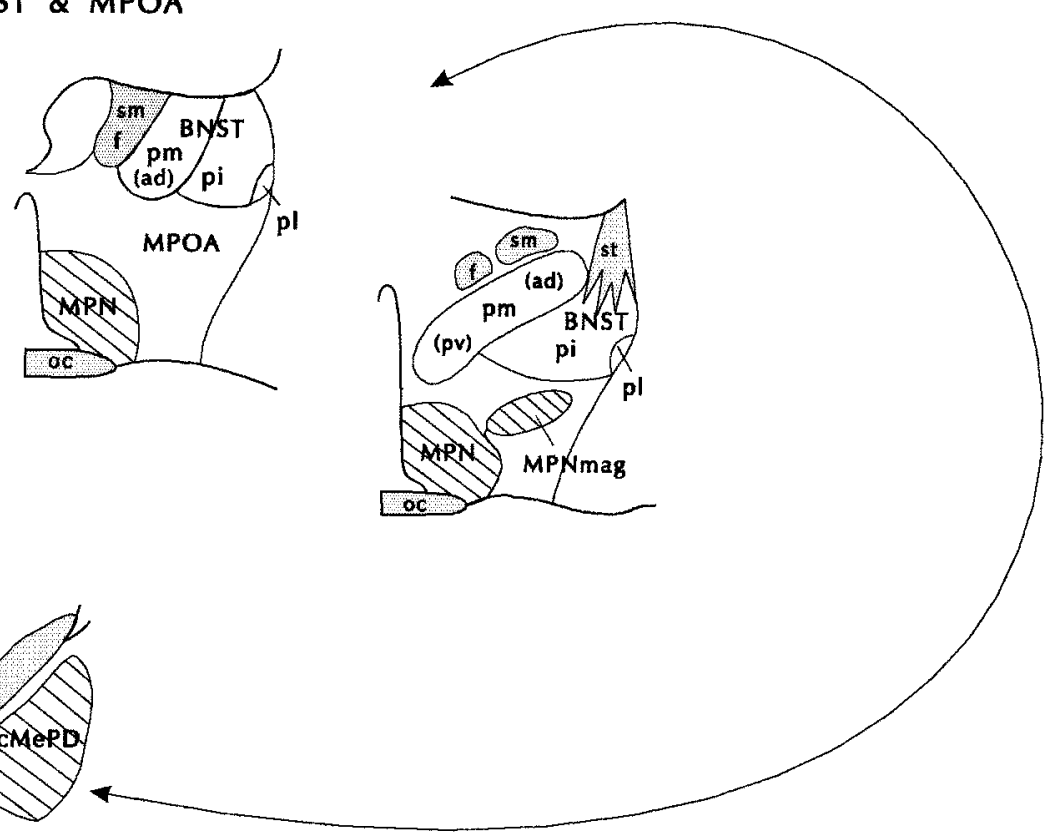

Figure 6 A summary of the net effect of experience on the pattern of Fos immunostaining within limbic regions of the male Syrian hamster brain. Significant activation above naive controls is illustrated with diagonal bars.

\section{Sexual Experience and the Effects of Repeated Handling}

Our results demonstrate significant elevations of Fos immunostaining within the caudal extension of the posterodorsal subdivision of the medial nucleus of the amygdala (cMePD), the MPN and the MPNmag of sexually experienced control males compared to sexually naive control males, when neither group had been with a female on the day of the experiment (Fig. 6). This experience-associated neuronal activation may reflect a conditioned response to one or more of the environmental cues associated with the three sexual experience pretests, such as the experimenter's presence, being handled and moved to the testing arena, or the testing arena itself.

The caudal MePD, MPN, and MPNmag belong to a larger circuit of neuroanatomically interconnected areas that receive chemosensory input from the vomeronasal organ (Davis et al., 1978; Kevetter and Winans, 1981; Maragos et al., 1989; Gomez and Newman, 1992) and contain dense populations of androgen-concentrating neurons, many of which are directly activated by mating behavior in this species (Wood and Newman, 1993). These three brain regions may represent the neuroanatomical substrate for previously documented behavioral changes, or learning, following sexual experience in the male hamster including a greater tendency to attempt intromissions following exposure to FHVS
(Macrides et al., 1977), an increase in the number of mounts and intromissions and the total time spent in copulation with a receptive female (Murphy, 1973), and decreased dependence upon stimulation from the vomeronasal organ (Meredith, 1986) and circulating gonadal steroids (Bunnell and Flesher, 1965, Bunnel and Kimmel, 1965; Lisk and Heimann, 1980) for the initiation of mating.

Alternatively, or in addition, the experience-dependent Fos production within this circuit may reflect activation of neurons that participate in neuroendocrine reflexes. Sexually experienced male rats, but not naive males, show consistent increases in plasma levels of luteinizing hormone and testosterone in response to sexually conditioned stimuli and in anticipation of sexual activity (Kamel and Frankel, 1978; Graham and Desjardins, 1980). A similar response to conditioned stimuli has not been demonstrated in the male hamster. However, sexually experienced males with lesions of the olfactory and vomeronasal systems show a significant increase in the levels of these two hormones in the presence of a receptive female, while sexually naive males do not (Pfeiffer and Johnston, 1994).

In our first study in this series, mating to multiple ejaculations (over a period of $45 \mathrm{~min}$ ) also produced significant activation of neurons within the anterior part of the medial amygdaloid nucleus (MeAD and MeAV), amygdalohippocampal area, posterome- 
dial cortical nucleus of the amygdala, and posterointermediate BNST in sexually naive male hamsters (Kollack and Newman, 1992). In our second study, these same structures plus the ventrolateral septum and PMV showed significant increases in the number of Fos-ir neurons above control after only 10 min of mating or intermale aggression in experimentally naive animals (Kollack-Walker and Newman, 1995). We interpreted the results of both studies to suggest that Fos production was enhanced in these areas as a result of processes common to social interactions, and not specific to the components of mating behavior such as intromissions and ejaculations. We further hypothesized that one or more of these areas might be coordinating general arousal in response to conspecific stimuli. Arousal, although common to a variety of situations, might be essential for the activation of more discrete circuits that drive specific responses appropriate to a given stimulus.

It was surprising to find in the present study that none of these brain areas showed increases in Fos expression in the experimental groups above control. Subsequent review of cell counts from individual animals in the control and experimental groups of the three studies indicated that males in both HCNAIVE and HC-EXP control groups in the present study had higher levels of Fos expression in many of these brain areas than had been observed in the control animals of our earlier studies, while the mated animals had levels that were consistent with what was observed previously in sexually active males (data not shown). Thus, the absence of significant increases in Fos expression within these brain areas following mating appears to result from enhanced Fos immunostaining in sexually experienced and repeatedly handled control animals, rather than a paucity of stimulated Fos production in behaviorally active males.

What might have caused increased Fos immunostaining within these brain areas in the control animals of the current study, i.e., in what ways did the handling of the control animals in these various studies differ? The previous studies had used experimentally naive control males that were either housed in groups and left in their home cage until they were anesthetized for perfusion (Kollack and Newman, 1992), or housed in isolation for 1 week prior to the experiment (a necessary protocol for reliably eliciting aggressive behavior), handled briefly, and then returned to their home cage for $60 \mathrm{~min}$ until they were anesthetized for perfusion (Kollack-Walker and Newman, 1995). In the present study, all males were group-housed, as in the first study (Kollack and Newman, 1992). These males, however, received three "pretests," handling experiences during which they were placed in an empty, clean testing arena (HC-NAIVE) for 10 min or in a testing arena with a receptive female (HC-EXP) until they achieved an ejaculation or 15 min had elapsed. On the final day of testing, each male was placed again into an empty, clean testing arena, where it remained for $1 \mathrm{~h}$ until being anesthetized for perfusion. Any of the stimuli associated with the three prior handling experiences, or being placed in a testing arena (rather than the home cage) for an hour before anesthetization, could have activated brain circuits involved in arousal and thus increased the Fos-ir in arousal circuits of both groups of control animals. Although group housing would have provided increased social stimulation that, in turn, could have increased Fos production in these circuits, this does not appear to be an important factor since the controls in our first study which were housed together both before and during the hour prior to being anesthetized actually showed less activation than those in the present study.

In summary, the results of this study suggest that cMePD, MPN, and MPNmag may contribute to an experience-based activation of sexual behavior and/ or neuroendocrine reflexes. In addition, MeAV and $\mathrm{MeAD}$, and possibly other brain regions, may respond in a more general way to changes in the animal's environment by increasing the animal's state of arousal, which is then followed by elicitation of context-specific behavioral responses. An inability of socially relevant cues, such as odors, to arouse an animal could explain the virtual elimination of sexual activity that is observed in male hamsters following bilateral damage to cells within the anterior parts of the medial amygdaloid nucleus (Lehman et al., 1980, 1983).

\section{Pheromones and Chemoinvestigatory Processes}

In the present study, sexually experienced males that investigated cotton swabs scented with FHVS showed a significant increase in Fos production in MePD, the anterior part of the posteromedial BNST [BNSTpm(ad)], and MPNmag (Fig. 7). This finding confirms the results reported by Fiber et al. (1993), in which they used male hamsters that had had prior sexual experience (personal communication). Similarly, exposure of sexually naive males to FHVS also results in the activation of neurons within MePD and BNSTpm(ad) [referred to as the caudal region of the posterior medial BNST, mBNSTpc (Fernandez-Fewell and Meredith, 1994)]. However, these latter authors did not report 


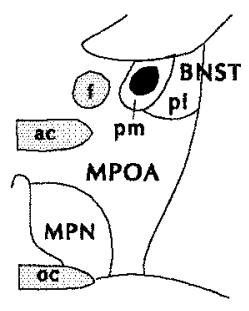

BNST \& MPOA
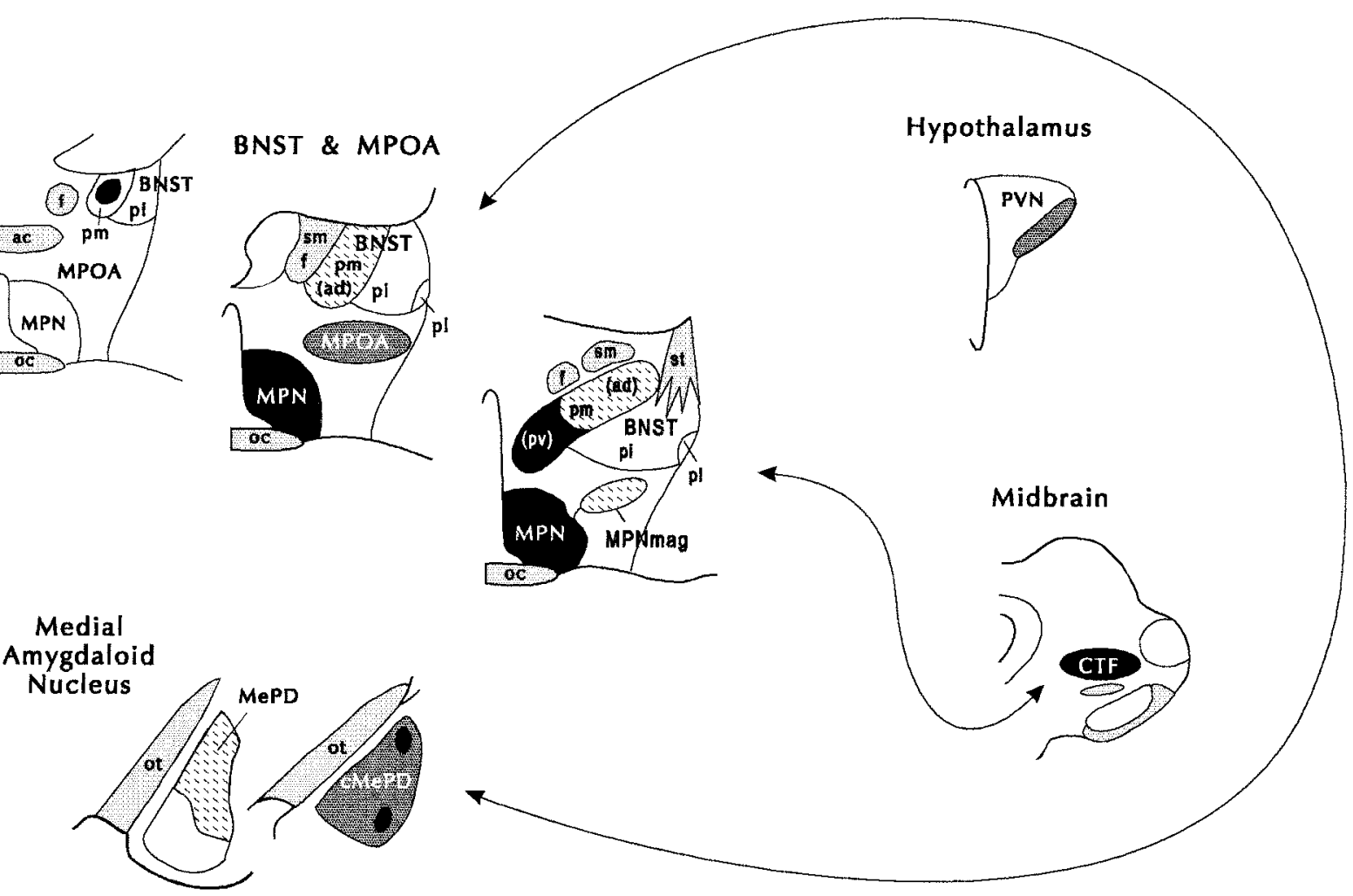

FHVS

Figure 7 A summary of the net effect of FHVS exposure and mating to ejaculation on Fos immunostaining within limbic regions of the male Syrian hamster brain. Significant activation above control after FHVS exposure is illustrated with stippling, after only one ejaculation with light gray, and after multiple ejaculations with black.

increased Fos expression in MPNmag of sexually naive males exposed to female pheromones.

In addition to pheromone-stimulated activation, Fos immunostaining increased further in BNST$\operatorname{pm}(\mathrm{ad})$ in males that mated to five or more ejaculations. It is presently unclear if this enhanced activation of neurons in BNSTpm(ad) reflects copulatory processes per se or the cumulative effects of investigating female odors over time.

The accessory olfactory system appears to be critical for stimulating Fos production in neurons within MePD and the anterodorsal level of BNSTpm following exposure to FHVS alone. Neurons within the posterodorsal Me and BNSTpm(ad) receive direct input from the accessory olfactory bulb (Scalia and Winans, 1975; Davis et al., 1978; Lehman and Winans, 1982), and bilateral removal of the vomeronasal organs, which provide input to the accessory olfactory bulbs, eliminates FHVS-mediated activation within these two brain regions in sexually naive male hamsters (Fernandez-Fewell and Meredith, 1994). In addition, neurons within the posterodorsal Me project centrally to BNSTpm and the MPOA (Gomez and Newman, 1992), providing an avenue for additional stimulation of neurons in BNSTpm and possibly for the excitation of neurons in MPNmag, although this latter area does not appear to be a direct target of MePD.

The FHVS-stimulated activation within MePD, BNSTpm(ad), and MPNmag may underlie the important effects of female pheromones on chemoinvestigatory and copulatory processes. Previous studies have shown that FHVS stimulates sexual attraction (chemoinvestigation) and initiation of mounting behavior (Lisk et al., 1972; Murphy, 1973; Darby et al., 1975; Gregory et al., 1975; Johnston, 1975; Powers et al., 1979) and distinct "attractant" and "mounting" pheromones isolated from FHVS have been implicated in mediating these two responses (Singer et al., 1976, 1984, 1986; Ma- 
crides et al., 1977; O'Connell et al., 1978; Clancy et al., 1984). Accordingly, lesions of the posterodorsal $\mathrm{Me}$ and the anterodorsal level of BNSTpm reduce anogenital chemoinvestigation (Lehman et al., 1983; Powers et al., 1987), while lesions including the medial aspect of MPNmag eliminate mounting, intromissions, and ejaculations (Powers et al., 1987).

Although exposure to FHVS may normally drive the male's chemoinvestigatory and mounting behavior, it is clear that other stimuli can serve a similar function, both behaviorally and in facilitating Fos production. Fernandez-Fewell and Meredith (1994) demonstrated that removal of the vomeronasal organs in sexually naive male hamsters eliminated Fos expression within BNSTpm in response to FHVS presented on glass slides. However, when similarly lesioned males were allowed to interact with receptive females, no decrement in Fos immunostaining was found, even when the behavior of these lesioned males involved only chemoinvestigation. This finding suggests that vomeronasal stimulation is not essential for the activation of neurons within BNSTpm, although such stimulation may play an important role in activating neurons in these areas under normal circumstances. Furthermore, it emphasizes the important role that this brain area plays in facilitating chemoinvestigatory behavior, a finding supported by the effect of lesions within BNST (Powers et al., 1987).

\section{Copulatory Processes}

In this study, mating to one or more ejaculations stimulated Fos expression in multiple brain areas, while mating to only five intromissions did not alter Fos immunolabeling in any of the brain areas examined. Following one ejaculation, the number of Fosir neurons increased within the cMePD, the dorsolateral MPOA, and the PVN, while significant elevations in Fos immunostaining were seen within the medial preoptic nucleus (MPN), the BNSTpm(pv), and the central tegmental field (CTF) only after multiple ejaculations (Fig. 7). In addition to these quantitative differences, qualitative changes were observed in the form of Fos positive cell clusters along the lateral border of $\mathrm{cMePD}$ and within the most rostral extent of posteromedial BNST (Fig. 7). These data confirm and extend previous reports in the hamster (Kollack and Newman, 1992; Fernandez-Fewell and Meredith, 1994; Wood and Newman, 1993; Kollack-Walker and Newman, 1995), rat (Robertson et al., 1991; Baum and Everitt, 1992; Wersinger et al., 1993; Coolen, 1995), mouse (Baum et al., 1994), and gerbil (Heeb and
Yahr, 1996) demonstrating the activation of neurons within specific limbic structures following male copulation.

The significance of a difference in the pattern of Fos immunolabeling following one or multiple ejaculations is presently unknown. In some brain regions, the activation of neurons during ejaculation may reflect a sequential process whereby additional Fos positive cells are recruited as the number of ejaculations increase. Indeed, in several of the areas activated after one ejaculation (i.e., cMePD and dorsolateral MPOA), there was a trend for more neurons to be present after five or more ejaculations. Likewise, in several of the areas activated only after five or more ejaculations [i.e., MPN and BNSTpm(pv)], there was a tendency for more neurons to be present after one ejaculation compared to the HC-EXP, FHVS, and 5-INTRO groups. A similar linear trend for increases in Fos immunostaining with greater amounts of male copulation have been described for cells in the MPOA of the male gerbil (Heeb and Yahr, 1996).

In other brain areas, differences in the pattern of Fos expression following one ejaculation and five ejaculations and long intromissions may reflect discrete changes in the physiologic state of the animal. For example, the activation of neurons after one ejaculation may reflect processes associated with the initiation of penile erection or seminal emission, while the activation of neurons at or just prior to the display of long intromissions might reflect processes associated with the termination of male copulation. An example of the latter process might be reflected in the formation of Fos-positive cell clusters in cMePD and BNSTpm, which were not seen in any male after only one ejaculation but could be detected in a subset of males after five ejaculations, and were present in all animals after long intromissions.

The MPOA is most consistently associated with male copulation. Within this region the dorsolateral area of the MPOA showed significant elevation of Fos-ir neurons after the first ejaculation in a series and the more medial MPN after multiple ejaculations. As in other vertebrates (reviewed in Meisel and Sachs, 1994), lesions of this region in the Syrian hamster eliminate male copulatory acts, including mounts, intromissions, and ejaculations (Eskes, 1984; Powers et al., 1987), but in the hamster such lesions can abolish copulation without altering chemoinvestigatory behavior (Powers et al., 1987). Thus, we hypothesize that activation of at least a subset of the Fos-ir neurons in this area is necessary for the animal to copulate. Precise localization of such a subset will require further study, but the 
effective lesions reported by Powers and colleagues (1987) were centered in the middle of the MPOA, including the lateral part of the MPN and the medial part of the MPNmag; lesions confined to the medial MPN or periventricular region in that study were ineffective (unpublished data). Interestingly, the magnocellular MPN, which lies just lateral to the MPN in the caudal MPOA region, was activated by exposure to female vaginal secretion alone. This pheromone-mediated activation of neurons within MPNmag may stimulate mounting behavior as exposure of males to FHVS elicits mounting (Singer et al., 1976, 1984, 1986; Macrides et al., 1977; O'Connell et al., 1978; Clancy et al., 1984) and lesions that damage the medial part of this nucleus eliminate mounting as well as subsequent components of copulation (Powers et al., 1987).

The most posterior part of the medial amygdala (cMePD), as well as the rostral posteromedial BNST (clusters) and the caudal BNSTpm(pv) also showed greater numbers of Fos-ir neurons in males that ejaculated, and like the MPN with which they are anatomically interconnected, these areas were activated in the late phase of sexual behavior, after multiple ejaculations. However, unlike lesions of the MPN, neither lesions placed within the posterodorsal Me (Lehman et al., 1983), nor small lesions of the BNST (Powers et al., 1987), eliminate copulation. In the male hamster, lesions of cMePD increase ejaculatory latency and alter the number of mounts and intromissions preceding the first ejaculation. Similarly, in the male rat, lesions that damage BNSTpm at its most rostral extent increase the number of intromissions preceding ejaculation as well as the length of time between intromissions (Emery and Sachs, 1976; Valcourt and Sachs, 1979). These findings suggest that neurons activated within cMePD and specific subregions of BNSTpm during mating may be involved in the temporal sequencing of male copulation.

In addition to a possible role in timing copulation, the caudal posterodorsal Me may participate in sexual satiety. Lesions of this area in female hamsters can produce a significant increase in the duration of copulation (Takahashi and Gladstone, 1988), and the appearance of neuronal cell clusters in cMePD was evident in all males who mated to long intromissions (indicating the onset of sexual satiety). Although these clusters were also present in several of the males who mated to only five ejaculations, it is unclear how many additional ejaculations these individuals would have shown before displaying long intromissions. In fact, one male in the Long-INTRO group had engaged in only six ejaculations prior to reaching satiety. Perhaps the clusters in cMePD represent "satiety centers" that play a role in terminating male copulation.

In the male rat, Baum and Everitt (1992) reported significant elevations in Fos-positive cells following intromissions alone in a specific region of the CTF, identified as the parvicellular subparafascicular nucleus of the thalamus (Faull and Mehler, 1985). However, Coolen (1995) observed significant elevations in Fos immunostaining within this region only in males that ejaculate. Furthermore, reducing the number of intromissions preceding an ejaculation did not affect Fos immunolabeling in neurons within this midbrain area, implying that ejaculation was the critical stimulus (Coolen, 1995). Mating to ejaculation has also been associated with Fos immunostaining in the CTF of male gerbils (Heeb and Yahr, 1996). In this study, neurons which we interpret to lie within the subparafascicular nucleus of the CTF in hamsters were activated in males that mated to five or more ejaculations. Although these data support the contention that cells within the CTF respond to ejaculatory stimuli, we cannot rule out the possibility that these cells are increasingly stimulated by multiple intromissions, since males in the 5-EJAC and Long-INTRO groups had more intromissions than males in the 5-INTRO and 1-EJAC groups.

The CTF has been implicated in mediating both motor and sensory aspects of male copulation. Electrolytic lesions placed within this region have been reported to eliminate all aspects of male copulation (Brackett and Edwards, 1984; Hansen and Gummesson, 1982), similar to the effects of lesions in the MPOA. Subsequently, it was shown that neurochemical lesions at this same level reduce the ability of a male to ejaculate but not the ability to mount or intromit (Hansen and Kohler, 1984), suggesting that cells within this area are important for ejaculation, while fibers that traversed the area are important for mounting and intromitting. More recently, it has been suggested that neurons within this midbrain locus process sensory information. Electrophysiological studies demonstrate that neurons within CTF are responsive to genital stimulation (Shimura and Shimokochi, 1990) and are active during male copulatory responses (Shimura and Shimokochi, 1991). Furthermore, bilateral transection of the pelvic nerves which carry sensory information into the spinal cord eliminates mating-induced Fos expression within the CTF (Wersinger et al., 1993). Baum and Everitt (1992) suggested that the CTF was processing genital somatosensory information associated with intromissions, because application of the anesthetic lidocaine to the male's penis prevented the male's intromissions and 
blocked the expression of Fos protein within the CTF. In contrast, Coolen (1995) suggested that the activation of neurons within this region following ejaculation may reflect genital visceral sensory information from the deep organs associated with the act of ejaculation, since somatosensory and visceral sensory information from the genitalia reach neurons within CTF. The decrease in Fos immunostaining in CTF following lidocaine treatment to the penis could be attributed to either superficial or deep sensory inputs, as both intromissions and ejaculations would be inhibited.

The processing of genital sensory information during copulation has been attributed not only to the CTF but also to the posterodorsal Me, BNSTpm, and MPN. In the female rat, the expression of Fos protein within Me, BNST, MPN, CTF, and other limbic structures is dependent upon sensory information carried by the pelvic nerves. Bilateral transection of the pelvic nerves results in elimination of Fos expression within all of these brain areas in the female (Wersinger et al., 1993). Based on the similarity of Fos immunostaining patterns seen in male and female rats following mating, it has been suggested that these brain regions in the male also reflect sensory processing. However, lesioning the pelvic nerve in the male results in a reduction of Fos immunolabeling only within the CTF (Wersinger et al., 1993). Additional studies will be necessary to determine if sensory information carried by the pudendal nerve is important for stimulating Fos expression within Me, BNST, and MPN in males following copulatory behavior, as suggested by Wersinger and colleagues (1993). The processing of genital sensory information might be important for the temporal sequencing of male copulation a role previously suggested for the posterodorsal $\mathrm{Me}$ and BNSTpm.

This study confirms our original observations of activation along the ventrolateral border of the PVN at a midrostrocaudal level in male hamsters following mating (Kollack and Newman, 1992; KollackWalker and Newman, 1995). Further, our results suggest that these cells may be involved in the process of ejaculation, although lesions of the PVN do not substantially alter copulatory behavior in the male hamster (Brown et al., 1988). Mating-induced activation within the PVN has also been observed in the males of other species, including rat (Baum and Everitt, 1992; Witt and Insel, 1994; Coolen, 1995 ) and mouse (Baum et al., 1994), but not in gerbil (Heeb and Yahr, 1996).

Witt and Insel (1994) reported mating-induced increases in Fos production in oxytocin-immunoreactive neurons within the lateral parvocellular re-

gion of the caudal PVN in male rats, a finding consistent with previous reports implicating oxytocin in male sex behavior (reviewed in Carter, 1992). However, Coolen (1995) did not observe colocalization of Fos protein and oxytocin in PVN neurons of male rats following mating. Similarly, the mating-specific group of activated neurons along the ventrolateral aspect of the hamster PVN does not appear to reflect the location of oxytocin-immunoreactive neurons based on the immunocytochemical localization of this peptide within the hamster PVN (Morin and Blanchard, 1993), and on preliminary colocalization studies with Fos and oxytocin in our laboratory (unpublished observations). Additional studies will be necessary to determine the neurochemical identity and behavioral role of these neurons in male hamster sexual behavior.

\section{CONCLUSIONS}

The expression of Fos protein following mating has made possible the identification of discrete subsets of neurons activated within individual brain nuclei and subnuclei following different amounts of sexual stimulation. Indeed, our results and those of other laboratories have demonstrated unique patterns of neuronal activation within adjacent regions of the same brain nucleus or subnucleus following chemoinvestigatory or copulatory stimuli. Analysis of cytoarchitectonic boundaries or the effects of lesions alone would not have suggested the presence of functionally distinct patterns of neuronal activation within a given brain region. In addition, our findings suggest that the pattern of activation along these mating behavior pathways in the hamster changes with different levels of sexual stimulation implicating sexual experience, investigation of female vaginal odors, and mating to ejaculation as relevant stimuli. Thus, Fos immunostaining can provide us with a view of the dynamic patterning of activation associated with increasing amounts of male copulation. These studies lay the foundation for future efforts to identify the sexually relevant neurochemical pathways embedded within these interconnected brain regions.

\section{ABBREVIATIONS}

ac

Aco

$\mathrm{AH}$ anterior commissure

anterior cortical nucleus of the amygdala anterior hypothalamic nucleus 


$\begin{array}{ll}\text { AHA } & \begin{array}{l}\text { amygdalohippocampal area } \\ \text { ARC }\end{array} \\ \text { arcuate nucleus of the hypothala- } \\ \text { mus } \\ \text { BNST } & \text { bed nucleus of the stria terminalis } \\ \text { BNSTam } & \text { BNST, anteromedial } \\ \text { BNSTal } & \text { anterolateral } \\ \text { BNSTav } & \text { BNST, anteroventral } \\ \text { BNSTpi } & \text { BNST, posterointermediate } \\ \text { BNSTpl } & \text { BNST, posterolateral } \\ \text { BNSTpm } & \text { BNST, posteromedial } \\ \text { BNSTpm (ad) } & \text { BNSTpm, anterodorsal } \\ \text { BNSTpm (pv) } & \text { BNSTpm, posteroventral } \\ \text { BLa } & \text { basolateral nucleus of the amyg- } \\ & \text { dala, anterior } \\ \text { cMePD } & \text { MePD, caudal extension } \\ \text { cp } & \text { cerebral peduncle } \\ \text { dPAG } & \text { periaqueductal gray, dorsal } \\ \text { f } & \text { fornix } \\ \text { IM } & \text { intercalated mass } \\ \text { LSv } & \text { ventral lateral septum } \\ \text { Me } & \text { medial nucleus of the amygdala } \\ \text { MeAD } & \text { Me, anterodorsal } \\ \text { MeAV } & \text { Me, anteroventral } \\ \text { MePD } & \text { Me, posterodorsal } \\ \text { MePV } & \text { Me, posteroventral } \\ \text { MPOA } & \text { medial preoptic area } \\ \text { MPN } & \text { medial preoptic nucleus } \\ \text { MPNmag } & \text { medial preoptic nucleus, magno- } \\ & \text { cellular } \\ \text { oc } & \text { optic chiasm } \\ \text { ot } & \text { optic tract } \\ \text { PLCo } & \text { posterolateral nucleus of the amyg- } \\ & \text { dala } \\ \text { PMCo } & \text { posteromedial nucleus of the amyg- } \\ & \text { dala } \\ \text { PMV } & \text { ventral premammillary nucleus } \\ \text { PVN } & \text { paraventricular nucleus of the hy- } \\ & \text { pothalamus } \\ \text { SCN } & \text { ventromedial nucleus of the hypo- } \\ \text { VMH } & \\ & \end{array}$

We thank Kaye Brabec, Sara Liliensiek, and Carrie Cartwright for valuable technical assistance. This work was supported by Grants NIH NS20629 (S.W.N.), P30HD-18258 (Morphology Core of the NIH-NICHD Center for the Study of Reproduction at the University of Michigan), and NIH DA07268-04 (S.K.W.).

\section{REFERENCES}

Baum, M. J., Brown, J. J. G., Kica, E., Rubin, B. S., Johnson, R. S., and PAPAIOANnOU, V. E. (1994). Ef- fect of a null mutation of the c-fos proto-oncogene on sexual behavior of male mice. Biol. Reprod. 50:10401048.

Baum, M. J., and EveritT, B. J. (1992). Increased expression of c-fos in the medial preoptic area after mating in male rats: role of afferent inputs from the medial amygdala and midbrain central tegmental field. Neuroscience 50:627-646.

Brackett, N. L., and Edwards, D. A. (1984). Medial preoptic connections with the midbrain tegmentum are essential for male sexual behavior. Physiol. Behav. 32:79-84.

Brown, M. H., Bandura, L. L., and Nunez, A. A. (1988). Axon-sparing lesions of the hypothalamic paraventricular nucleus abolish gonadal responses to photoperiod in male Syrian hamsters. J. Biol. Rhythms 3:59-69.

Brown, E. E., Robertson, G. S., and Fibiger, H. C. (1992). Evidence for conditional neuronal activation following exposure to a cocaine-paired environment: role of forebrain limbic structures. J. Neurosci. 12:4112-4121.

Bunnell, B. N., and Flesher, C. K. (1965). Copulatory behavior of male hamsters as a function of time since androgen withdrawal. Psychon. Sci. 3:181-182.

Bunnell, B. N., and Kimmel, M. E. (1965). Some effects of copulatory experience on postcastration mating behavior in the male hamster. Psychon. Sci. 3:179180.

Bunnell, B. N., Boland, B. D., and Dewsbury, D. A. (1977). Copulatory behavior of golden hamsters (Mesocricetus auratus). Behaviour 61:180-206.

CARTER, C. S. (1992). Oxytocin and sexual behavior. Neurosci. Biobehav. Rev. 16:131-144.

Clancy, A. N., Macrides, F., Singer, A. G., and Agosta, W. C. (1984). Male hamster copulatory responses to a high molecular weight fraction of vaginal discharge: effects of vomeronasal removal. Physiol. Behav. 33:653-660.

Coolen, L. M. (1995). The neural organization of sexual behavior in the male rat: a functional neuroanatomic Fos-study. Doctoral dissertation, Katholieke Universiteit Nijmegen, The Netherlands.

Darby, E. M., Devor, M., and Chorover, S. L. (1975). A presumptive sex pheromone in the hamster: some behavioral effects. J. Comp. Physiol. Psychol. 88:496502.

Davis, B. J., Macrides, F., Young, W. M., Schneider, S. P., and Rosene, D. L. (1978). Efferents and centrifugal afferents of the main and accessory olfactory bulbs in the hamster. Brain Res. Bull. 3:59-72.

De Jonge, F. H., Oldenburger, W. P., Louwerse, A. L., and VAN DE Poll, N. E. (1992). Changes in male copulatory behavior after sexual exciting stimuli: effects of medial amygdala lesions. Physiol. Behav. 52:327-332.

Dragunow, M., and Faull, R. (1989). The use of c-fos as a metabolic marker in neuronal pathways tracing. $J$. Neurosci. Methods 29:261-265.

EMERY, D. E., and SACHS, B. D. (1976). Copulatory be- 
havior in male rats with lesions in the bed nucleus of the stria terminalis. Physiol. Behav. 17:803-806.

EsKes, G. A. (1984). Neural control of the daily rhythm of sexual behavior in the male golden hamster. Brain Res. 293: $127-144$.

FAULl, R. L., and MEHLER, W. R. (1985). Thalamus. In: The Rat Nervous System. Vol. 1. Paxinos, G., Ed. Academic Press, New York, pp. 129-168.

Fernandez-Fewell, G. D., and Meredith, M. (1994). c-fos expression in vomeronasal pathways of mated or pheromone-stimulated male golden hamsters: contributions from vomeronasal sensory input and expression related to mating performance. J. Neurosci. 14:36433654.

Fiber, J. M., Adames, P., and Swann, J. M. (1993). Pheromones induce c-fos in limbic areas regulating male hamster mating behavior. Neuroreport 4:871874.

Gomez, D. M., and Newman, S. W. (1992). Differential projections of the anterior and posterior regions of the medial amygdaloid nucleus in the Syrian hamster. $J$. Comp. Neurol. 317:195-218.

Graham, J. M., and Desjardins, C. (1980). Classical conditioning: induction of luteinizing hormone and testosterone secretion in anticipation of sexual activity. Science 210:1039-1041.

Gregory, E., Engel, K., and Pfaff, D. (1975). Male hamster preference for odors of female hamster vaginal discharges: studies of experiential and hormonal determinants. J. Comp. Physiol. Psychol. 89:442-446.

Hansen, S., and Gummesson, B. M. (1982). Participation of the lateral midbrain tegmentum in the neuroendocrine control of sexual behavior and lactation in the rat. Brain Res. 251:319-325.

Hansen, S., and KoHler, C. (1984). The importance of the peripeduncular nucleus in the neuroendocrine control of sexual behavior and milk ejection in the rat. Neuroendocrinology 39:563-572.

HeEB, M. M., and YAHR, P. (1996). c-fos immunoreactivity in the sexually dimorphic area of the hypothalamus and related brain regions of male gerbils after exposure to sex-related stimuli or performance of specific sexual behaviors. Neuroscience. In press.

Hsu, S.-M., RaInE, L., and FANGER, H. (1980). The use of avidin-biotin-peroxidase complex $(\mathrm{ABC})$ in immunoperoxidase techniques: a comparison between $\mathrm{ABC}$ and unlabeled antibody (PAP) procedures. J. Histochem. Cytochem. 29:577-580.

Hsu, S.-M., and Soban, E. (1982). Color modification of diaminobenzidine (DAB) precipitation by metallic ions and its application for double immunohistochemistry. J. Histochem. Cytochem. 30:1079-1082.

Hunt, S. P., Pini, A., and Evan, G. (1987). Induction of c-fos-like protein in spinal cord neurons following sensory stimulation. Nature 328:632-634.

Johnston, R. E. (1974). Sexual attraction function of golden hamster vaginal secretion. Behav. Biol. 12:111117.

Johnston, R. E. (1975). Sexual excitation function of hamster vaginal secretion. Anim. Learn. Behav. 3:161166.

Kamel, F., and Frankel, A. I. (1978). Hormone release during mating in the male rat: time course, relation to sexual behavior, and interaction with handling procedures. Endocrinology 103:2172-2179.

Kevetter, G. A., and Winans, S. S. (1981). Connections of the corticomedial amygdala in the golden hamster. I. Efferents of the "vomeronasal amygdala." $J$. Comp. Neurol. 197:81-98.

Kollack, S. S., and Newman, S. W. (1992). Mating behavior induces selective expression of Fos protein within the chemosensory pathways of the male Syrian hamster brain. Neurosci. Lett. 143:223-228.

Kollack-Walker, S., and Newman, S. W. (1995). Mating and agonistic behavior produce different patterns of Fos immunolabeling in the male Syrian hamster brain. Neuroscience 66:721-736.

Lehman, M. N., Powers, J. B., and Winans, S. S. (1983). Stria terminalis lesions alter the temporal pattern of copulatory behavior in the male golden hamster. Behav. Brain Res. 8:109-128.

Lehman, M. N., and Winans, S. S. (1982). Vomeronasal and olfactory pathways to the amygdala controlling male hamster sexual behavior: autoradiographic and behavioral analyses. Brain Res. 240:27-41.

Lehman, M. N., Winans, S. S., and Powers, J. B. (1980). Medial nucleus of the amygdala mediates chemosensory control of male hamster sexual behavior. Science 210:557-560.

LisK, R. D., and HEIMANN, J. (1980). The effects of sexual experience and frequency of testing on retention of copulatory behavior following castration in the male hamster. Behav. Neural Biol. 28:156-171.

Lisk, R. D., Zeiss, J., and Ciaccio, L. A. (1972). The influence of olfaction on sexual behavior in the male golden hamster (Mesocricetus auratus). J. Exp. Zool. 181:69-78.

Macrides, F., Johnson, P. A., and Schneider, S. P. (1977). Responses of the male golden hamster to vaginal secretion and dimethyl disulfide: attraction versus sexual behavior. Behav. Biol. 20:377-386.

Maragos, W. F., Newman, S. W., Lehman, M. N., and Powers, J. B. (1989). Neurons of origin and fiber trajectory of amygdalofugal projections to the medial preoptic area in Syrian hamsters. J. Comp. Neurol. 280:59-71.

Meisel, R. L., and SACHS, B. D. (1994). The physiology of male sexual behavior. In: The Physiology of Reproduction. E. Knobil and J. Neill, Eds. Raven Press, New York, pp. 3-105.

Meredith, M. (1986). Vomeronasal organ removal before sexual experience impairs male hamster mating behavior. Physiol. Behav. 36:737-743.

Morgan, J. I., Cohen, D. R., Hempstead, J. L., and CuRRAN, T. (1987). Mapping patterns of c-fos expression in the central nervous system after seizure. Science 237:192-197.

Morin, L. P., and Blanchard, J. (1993). Organization 
of the hamster paraventricular hypothalamic nucleus. J. Comp. Neurol. 332:341-357.

MurPhy, M. R. (1973). Effects of female hamster vaginal discharge on the behavior of male hamsters. Behav. Biol. 9:367-375.

O'Connell, R. J., Singer, A. G., Macrides, F., PfaffManN, C., and Agosta, W. C. (1978). Responses of the male golden hamster to mixtures of odorants identified from vaginal discharge. Behav. Biol. 24:244-255.

Pezzone, M. A., Wen-Sen, L., Hoffman, G. E., and RABIN, B. S. (1992a). Induction of c-Fos immunoreactivity in the rat forebrain by conditioned and unconditioned aversive stimuli. Brain Res. 597:41-50.

Pezzone, M. A., Wen-Sen, L., Hoffman, G. E., PezZONE, K. M., and RABIN, B. S. (1992b). Activation of brainstem catecholaminergic neurons by conditioned and unconditioned aversive stimuli revealed by c-Fos immunoreactivity. Brain Res. 608:310-318.

Pfeiffer, C. A., and Johnston, R. E. (1994). Hormonal and behavioral responses of male hamsters to females and female odors: roles of olfaction, the vomeronasal system, and sexual experience. Physiol. Behav. 55:129-138.

Powers, J. B., Fields, R. B., and Winans, S. S. (1979). Olfactory and vomeronasal system participation in male hamsters' attraction to female vaginal secretions. Physiol. Behav. 22:77-84.

Powers, J. B., Newman, S. W., and Bergondy, M. L. (1987). MPOA and BNST lesions in male Syrian hamsters: differential effects on copulatory and chemoinvestigatory behaviors. Behav. Brain Res. 23:181-195.

Robertson, G. S., Pfaus, J. G., Atkinson, L. J., MatsuMURA, H., Phillips, A. G., and Fibiger, H. C. (1991). Sexual behavior increases c-fos expression in the forebrain of the male rat. Brain Res. 564:352-357.

Sagar, S. M., Sharp, F. R., and Curran, T. (1988). Expression of c-fos protein in brain: metabolic mapping at the cellular level. Science 240:1328-1331.

Scalia, F., and Winans, S. S. (1975). The differential projections of the olfactory bulb and accessory olfactory bulb in mammals. J. Comp. Neurol. 161:31-56.

Shimura, T., and SHIMокосHI, M. (1990). Involvement of the lateral mesencephalic tegmentum in copulatory behavior of male rats: neuron activity in freely moving animals. Neurosci. Res. 9:173-183.

Shimura, T., and Shimokochi, M. (1991). Modification of male rat copulatory behavior by lateral midbrain stimulation. Physiol. Behav. 50:989-994.

Singer, A. G., Clancy, A. N., Macrides, F., and
AgostA, W. C. (1984). Chemical studies of hamster vaginal discharge: male behavioral responses to a high molecular weight fraction require physical contact. Physiol. Behav. 33:645-651.

Singer, A. G., O'Connell, R. J., Macrides, F., BeneSath, A. F., and Agosta, W. C. (1976). Dimethyl disulfide: an attractant pheromone in hamster vaginal secretion. Science 191:948-950.

Singer, A. G., Macrides, F., Clancy, A. N., and Agosta, W. C. (1986). Purification and analysis of a proteinaceous aphrodisiac pheromone from hamster vaginal discharge. J. Biol. Chem. 261:13323-13326.

SWANK, M. W., and BERnSTEIn, I. L. (1994). c-Fos induction in response to a conditioned stimulus after single trial taste aversion learning. Brain Res. 636:202208.

TAKahashi, L. K., and Gladstone, C. D. (1988). Medial amygdaloid lesions and the regulation of sociosexual behavioral patterns across the estrous cycle in female golden hamsters. Behav. Neurosci. 102:268-275.

Valcourt, R. J., and SAchs, B. D. (1979). Penile reflexes and copulatory behavior in male rats following lesions in the bed nucleus of the stria terminalis. Brain Res. Bull. 4:131-133.

van Straaten, F., Muller, R., Curran, T., van BeVEREN, C., and Verma, I. M. (1983). Nucleotide sequence of a human c-onc gene: deduced amino acid sequence of human c-fos protein. Proc. Natl. Acad. Sci. USA 80:3183-3187.

Wersinger, S. R., Baum, M. J., and Erskine, M. S. (1993). Mating-induced FOS-like immunoreactivity in the rat forebrain: a sex comparison and a dimorphic effect of pelvic nerve transection. J. Neuroendocrinol. 5:557-568.

WitT, D. M., and InsEL, T. R. (1994). Increased Fos expression in oxytocin neurons following masculine sexual behavior. J. Neuroendocrinol. 6:13-18.

Wood, R. I., and Newman, S. W. (1993). Mating activates androgen receptor-containing neurons in chemosensory pathways of the male Syrian hamster brain. Brain Res. 614:65-77.

Zamble, E., Hadad, G. M., Mitchell, J. B., and CutMORE, T. R. H. (1985). Pavlovian conditioning of sexual arousal: first-order and second-order effects. J. Exp. Psychol. Anim. Behav. 11:598-610.

Zamble, E., Mitchell, J. B., and Findlay, H. (1986). Pavlovian conditioning of sexual arousal: parametric and background manipulations. J. Exp. Psychol. Anim. Behav. 12:403-411. 